\author{
Review
}

\title{
Recent studies on reaction pathways and applications of sugar orthoesters in synthesis of oligosaccharides
}

\author{
Fanzuo Kong* \\ Research Center for Eco-Environmental Sciences, Chinese Academy of Sciences, Beijing 100085, China \\ Received 9 August 2006; accepted 29 September 2006 \\ Available online 5 October 2006 \\ Dedicated to the memory of Professor Nikolay K. Kochetkov
}

\begin{abstract}
Formation of sugar-sugar orthoesters consisting of a fully acylated mono- or disaccharide donor and a partially protected mono- or disaccharide acceptor is regioselective, and rearrangement of the orthoesters via RO-(orthoester)C bond cleavage gives a dioxolenium ion intermediate leading to 1,2-trans glycosidic linkage. The activity order of hydroxyl groups in the partially protected mannose and glucose acceptors is $6-\mathrm{OH}>3-\mathrm{OH}>2$ - or $4-\mathrm{OH}$. The coupling reactions with acylated glycosyl trichloroacetimidates as the donors usually give orthoesters as the intermediates specially when the coupling is carried out at slowed rates, and this is successfully used in regio- and stereoselective syntheses of oligosaccharides. Mannose and rhamnose orthoesters readily undergo O-2-(orthoester)C bond breaking, and this is used for synthesis of $\alpha-(1 \rightarrow 2)$-linked oligosaccharides. ( $1 \rightarrow 3)$-Glucosylation is special since the rearrangement of its sugar orthoester intermediates can occur with either RO-(orthoester)C bond cleavage with formation of the dioxolenium ion leading to 1,2-trans linkage, or C-1-O-1 bond cleavage leading to 1,2-cis linkage, and this is dependent upon the structures of donor and acceptor that compose the orthoester.
\end{abstract}

(c) 2006 Elsevier Ltd. All rights reserved.

Keywords: Orthoester; Rearrangement; Oligosaccharide synthesis

\section{Contents}

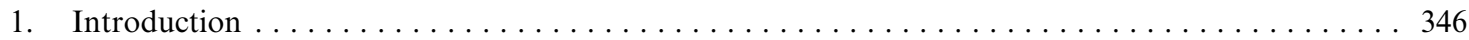

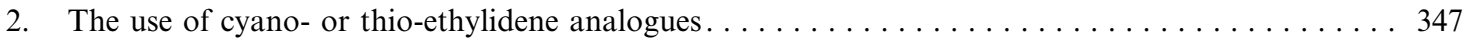

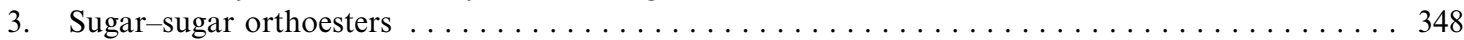

3.1. Preparation and identification of sugar-sugar orthoesters . . . . . . . . . . . . . . 349

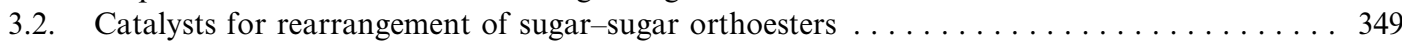

3.3. Regio- and stereoselective synthesis of oligosaccharides via sugar-sugar orthoester intermediates 349

3.3.1. Use of unprotected or partially protected glucosides in regio- and stereoselective

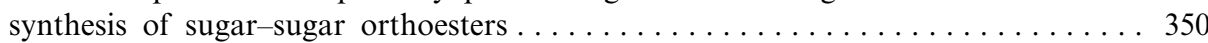

3.3.2. Use of unprotected or partially protected mannosides in regio- and stereoselective

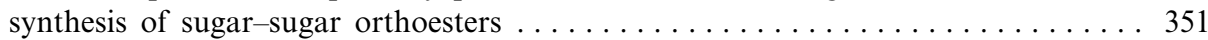

3.3.3. Use of unprotected rhamnoside in regio- and stereoselective synthesis of sugar-

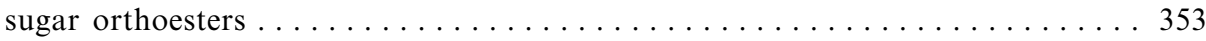

3.3.4. Possible mechanism of sugar-sugar orthoester rearrangement . . . . . . . . . . . 353

3.4. Application of sugar-sugar orthoester formation-rearrangement in oligosaccharide synthesis 353

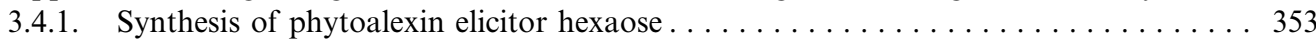

3.4.2. Synthesis of $\alpha-(1 \rightarrow 6)$-linked manno- and $\beta-(1 \rightarrow 6)$-linked gluco- and galacto-

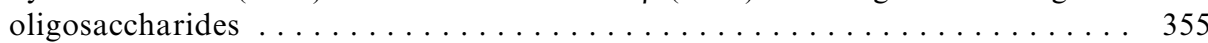

\footnotetext{
*Tel.: +86 10 64843715; fax: +86 1062923563 ; e-mail: fzkong@mail.rcees.ac.cn
} 
3.4.3. Synthesis of 1,1 -linked oligosaccharides $\ldots \ldots \ldots \ldots \ldots 5$

3.5. Self-condensation of mannose 1,2-allyl orthoester for preparation of $(1 \rightarrow 2)$-linked

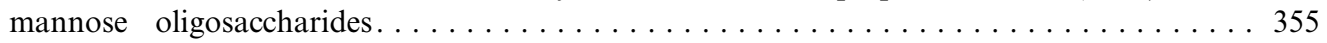

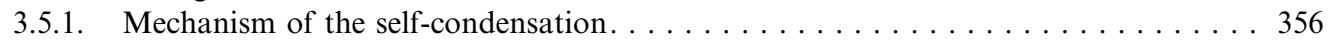

3.5.2. Self-condensation of rhamnose 1,2-allyl orthoester for preparation of

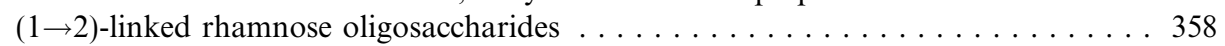

3.5.3. Application of self-condensation of mannose and rhamnose orthoesters . . . . . 358

3.5.4. A typical example for the use of O-2-(orthoester) $\mathrm{C}$ bond cleavage of

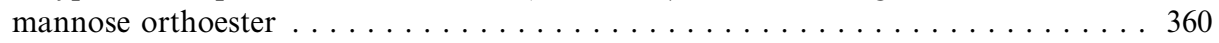

3.6. Regio- and stereoselective synthesis of oligosaccharides with sugar trichloroacetimidates as the donors and unprotected or partially protected glycosides as the acceptors via

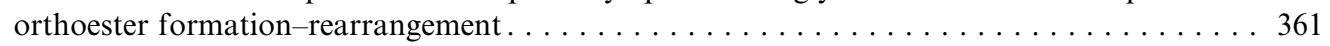

3.6.1. Sugar-sugar orthoester is usually the intermediate of glycosylation with sugar

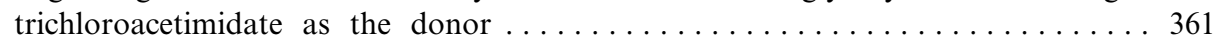

3.6.2. Application of glycosyl trichloroacetimidate donors in regio- and stereoselective synthesis of biologically important oligosaccharides . . . . . . . . . . . . . . . 364

3.6.3. Sugar-sugar orthoester is not always convertible to the desired oligosaccharide . . 366

3.7. Rearrangement of sugar-sugar orthoesters leading to 1,2 -cis-glycosidic linkage . . . . . . . 367

3.7.1. Coupling of acylated $\beta-(1 \rightarrow 3)$-linked glucobiosyl trichloroacetimidate donor with $3^{\prime}$-unprotected acylated $\beta$ - $(1 \rightarrow 3)$-linked glucobiosyl acceptor gives pure $\alpha$-linked

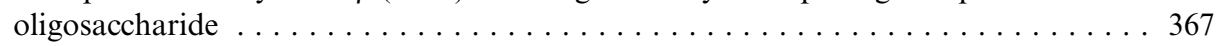

3.7.2. Sugar-sugar orthoester is the intermediate in $(1 \rightarrow 3)$-glucosylation capable of producing $\alpha$-linked oligosaccharide through intramolecular rearrangement . . . . 368

3.7.3. Application of the orthoester intramolecular rearrangement for synthesis of

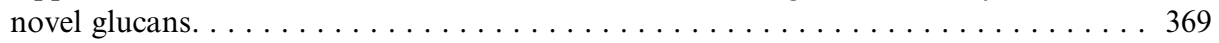

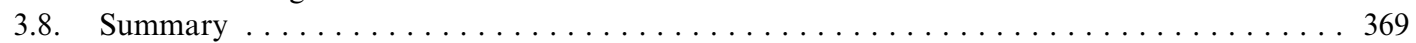

References .................................... 372

\section{Introduction}

Carbohydrate 1,2-orthoesters have long been known ${ }^{1}$ and have been used for the synthesis of 1,2-trans-glycosides. $^{2}$ Kochetkov and his coworkers ${ }^{3}$ were the first to utilize sugar 1,2-orthoesters in di- and trisaccharide syntheses. A considerable number of studies ${ }^{4 a-h}$ were focused on the products and mechanisms of the acidcatalyzed reactions of sugar orthoesters in a variety of systems. In spite of that the studies on the pathways of orthoesters reactions in different groups give different issues, we can now outline the reaction pathways of orthoesters as indicated in Chart 1.

Pathway (a) starts from coordination of the O-1 of orthoester 1 with a Lewis acid followed by the C-1-O-1 bond breaking giving an intermediate 2 containing a delocalized carbon cation. Attacking the $\mathrm{C}-1$ of $\mathbf{2}$ from either side by an alkoxide $\mathrm{R}^{\prime \prime} \mathrm{O}^{-}$is accompanied by liberation of an ester RCOOR', giving a glycoside $\mathbf{3}$ with mixed anomeric configuration and a $\mathrm{C}-2$ free hydroxyl group. Alternatively, intramolecular rearrangement of 2 stereospecifically yields a 1,2 -cis-linked $\alpha$-glycoside $\mathbf{4}$ with $\mathrm{R}^{\prime}$ as the aglycon. Besides, intermolecular rearrangement of $\mathbf{2}$ produces a carbocation $\mathbf{5}$ as the intermediate that reacts with $\mathrm{R}^{\prime} \mathrm{O}^{-}$and $\mathrm{R}^{\prime \prime} \mathrm{O}^{-}$to yield 2- $O$-acylated glycosides 6 and 7, respectively, as anomeric mixtures.

Pathway (b) starts from coordination of the $\mathrm{O}$ of $\mathrm{OR}^{\prime}$ in orthoester $\mathbf{1}$ with a Lewis acid followed by the
$\mathrm{C}$ (orthoester)-OR' bond cleavage producing a dioxolenium ion $\mathbf{9}$ that reversibly transforms either to $\mathbf{5}$ or via transorthoesterification ${ }^{4 f, g}$ to a new orthoester 8 . The orthoester $\mathbf{8}$, like the original orthoester $\mathbf{1}$, can also undergo reactions through pathways $(\mathrm{a}-\mathrm{d})$. The reactive cation 9 reacts with either $\mathrm{R}^{\prime \prime} \mathrm{O}^{-}$or $\mathrm{R}^{\prime} \mathrm{O}^{-}$, which attacks the $\mathrm{C}-1$ of 9 in an $\mathrm{S}_{\mathrm{N}} 2$ mode, to yield 1,2-trans-linked glycoside 10 or 11 , respectively.

Pathway (c) starts from coordination of the O-1 of orthoester 1 with a Lewis acid followed by the C(orthoester)-O-1 bond cleavage to give a reactive intermediate 12. Elimination of $\mathrm{RCOOR}^{\prime}$ produces 1,2-diol 13, while elimination of $\mathrm{R}^{\prime} \mathrm{OH}$ affords $\mathbf{1 4}$ with hydroxyl at $\mathrm{C} 1$ and 2-O-acyl group. Because of no formation of the desired glycoside, pathway (c) is usually considered as an undesired side reaction. ${ }^{5 a-d}$

Pathway (d) begins with coordination of the $\mathrm{O}-2$ of orthoester 1 with a Lewis acid followed by the O-2$\mathrm{C}$ (orthoester) bond cleavage, and the resultant reactive intermediate 15 reacts with $\mathrm{R}^{\prime \prime} \mathrm{OH}$ to give a 1,2-translinked glycoside 16 with C-2-free hydroxyl group. This kind of reaction is only observed on mannose derivatives, and is also considered as a side reaction because of the low yield. ${ }^{5 e}$

As shown in Chart 1 and described above, several pathways are available for the glycosylation with simple orthoesters as the glycosyl donors, and in some cases, side reactions are not avoided. Attempts to minimize 


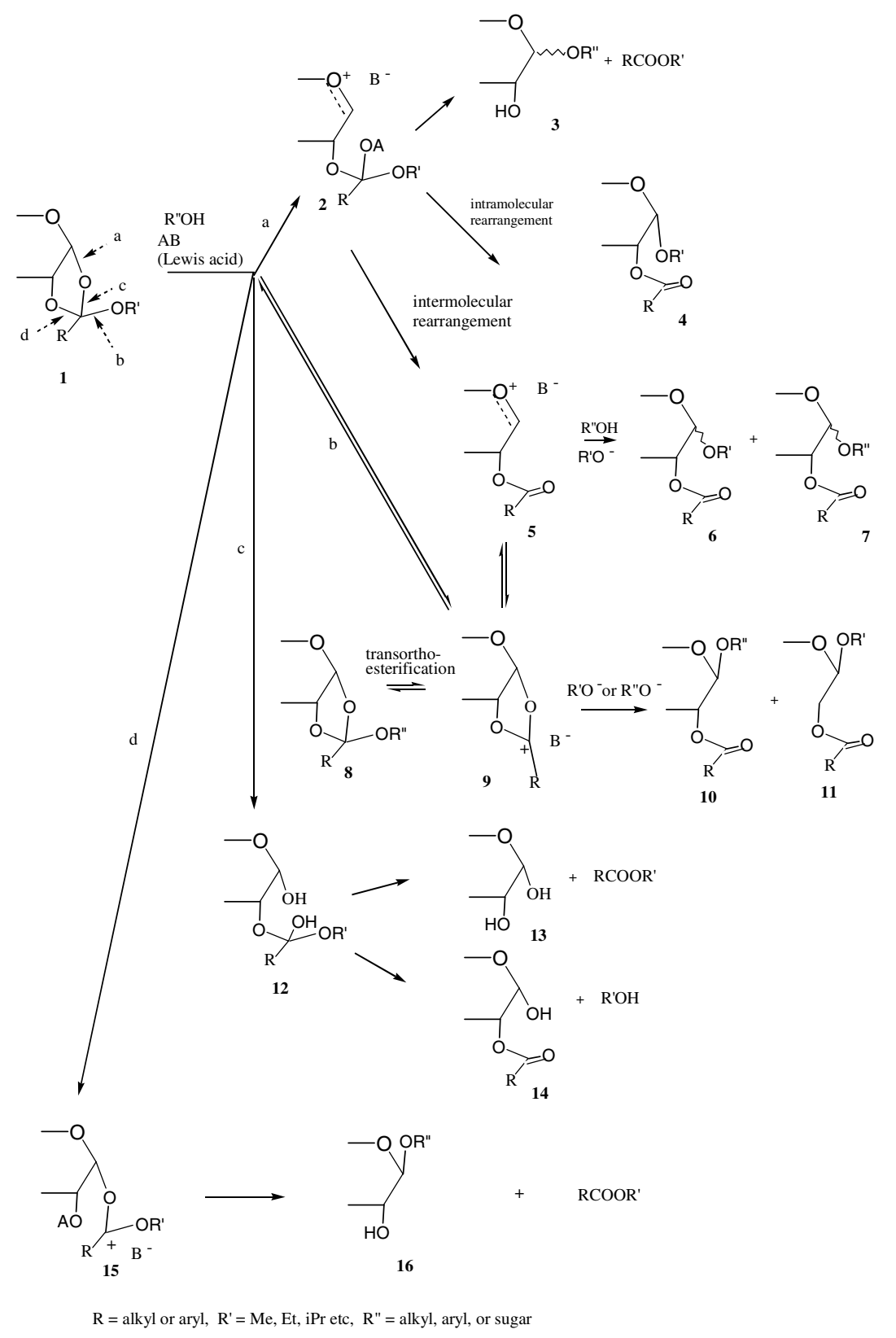

Chart 1. Possible reaction pathways of sugar orthoesters.

these undesirable side reactions have included the replacement of protic acids by Lewis acids such as mercuric bromide and cyanide, and the use of orthoesters derived from alcohols that are poor nucleophiles and have low boiling points such that they can be removed from the reaction by distillation. ${ }^{4 \mathrm{e}}$ However, these improvements can not completely overcome the inherent shortcomings of the method. Thus, glycosylation with simple orthoesters as the glycosyl donors has no longer been a good choice for oligosaccharide synthesis especially compared to latter developed techniques such as the methods with glycosyl trichloroacetimidate ${ }^{6}$ and thioglycoside donors. ${ }^{7}$

\section{The use of cyano- or thio-ethylidene analogues}

The most dramatic improvements for orthoester method came from the use of cyano- or thio-ethylidene analogues ${ }^{4 \mathrm{e}}$ as glycosylation agents, and Kochetkov's group have made great contributions. ${ }^{8}$ In this method, trityl perchlorate is used as the catalyst and the acceptor hydroxyl compounds present as their trityl ethers. This leads to the preferential cleavage of the cyano or thio group by the trityl cation, as depicted in Scheme 1 .

Reaction of the cyano-ethylidene analogue 17 with the acceptor 19 with 6-O-trityl group in the presence of $\mathrm{TrClO}_{4}$ produces a $(1 \rightarrow 6)$-linked disaccharide 20 with 
1,2-trans-configuration. In the condensation, no water molecule is released while it is known that water is harmful for coupling reactions. A further advantage of this method is that only catalytic amounts of trityl perchlorate are necessary since the reagent is regenerated in the process of the reaction. ${ }^{4 \mathrm{e}}$

This method is also used for synthesis of $(1 \rightarrow 3)$ - or $(1 \rightarrow 4)$-linked disaccharide, for example, coupling of $\mathbf{1 7}$ with 21 gives $(1 \rightarrow 3)$ - $\beta$-linked disaccharide $\mathbf{2 2}$, while condensation of $\mathbf{2 3}$ with $\mathbf{2 4}$ gives $(1 \rightarrow 4)-\beta$-linked disaccharide 25. However, the stereoselectivity is not as good as the one in $(1 \rightarrow 6)$-linked disaccharide synthesis since some $\alpha$-linked byproducts are obtained ${ }^{9 \mathrm{a}}$ as shown in Scheme 1. To improve the stereoselectivity, special reaction conditions such as high pressure and special catalysts are needed..$^{\mathrm{g}, \mathrm{c}}$

When the cyano-ethylidene and trityl groups are in the same molecule, condensation polymerization can occur. For example, condensation of 6-O-tritylated 1,2-O-cyanoethylidene-D-glucose (26), -D-galactose (27), and -D-mannose (28) produces $(1 \rightarrow 6)-\beta$-linked glucan (29), ${ }^{10 \mathrm{a}}$ galactan (30), ${ }^{10 \mathrm{~b}}$ and $-\alpha$-linked mannan (31), ${ }^{10 \mathrm{c}}$ respectively, as shown in Scheme 2. Oligosaccharides thus obtained have a degree of polymerization of
10-20, a wide distribution of molecular weights that is usually observed in stepwise polymerization.

A great advantage of this method is that polymers consisting of complex oligosaccharide units can be readily obtained, For instance, polymerization of disaccharide unit $\mathbf{3 2}$ yields a polymer that is composed of the disaccharide repeating unit ${ }^{11}$ as indicated in Scheme 2. Polysaccharides containing more complex oligosaccharide repeating units can also be synthesized in this way. A detailed and comprehensive review regarding this method has been contributed by Kochetkov. ${ }^{8}$ Although this method is much better than the ones with simple orthoesters as the donors, the use of highly toxic $\mathrm{NaCN}$ reagent in the preparation of the starting materials limits its application.

\section{Sugar-sugar orthoesters}

Complex sugar orthoesters containing sugar derivatives as alkoxide units are the so called 'sugar-sugar' orthoesters. Compared to simple sugar orthoesters, the reaction pathways of sugar-sugar orthoesters are relatively simplified since the consideration is mainly focused on
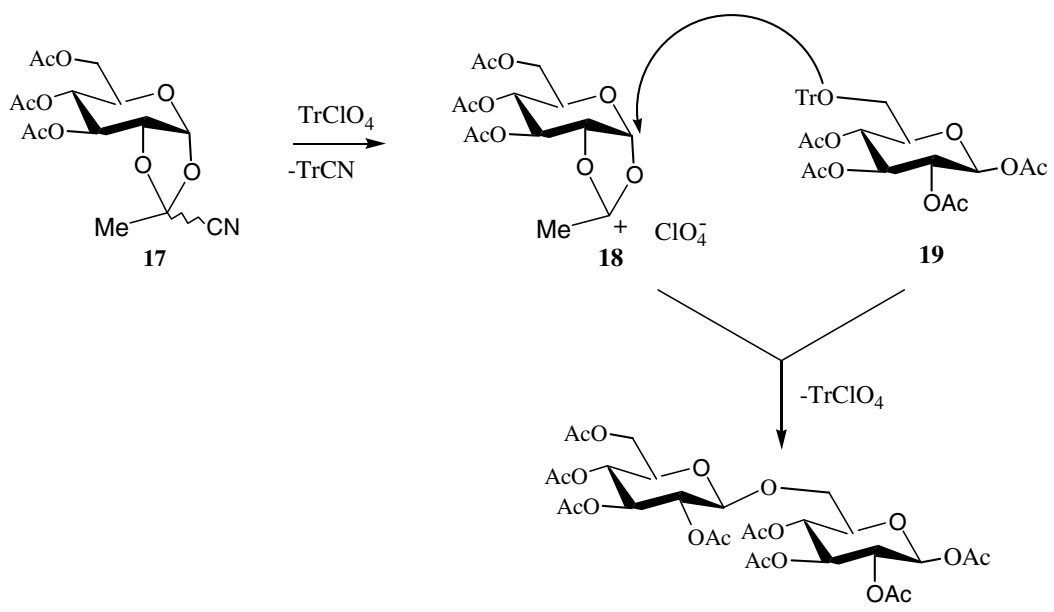

20
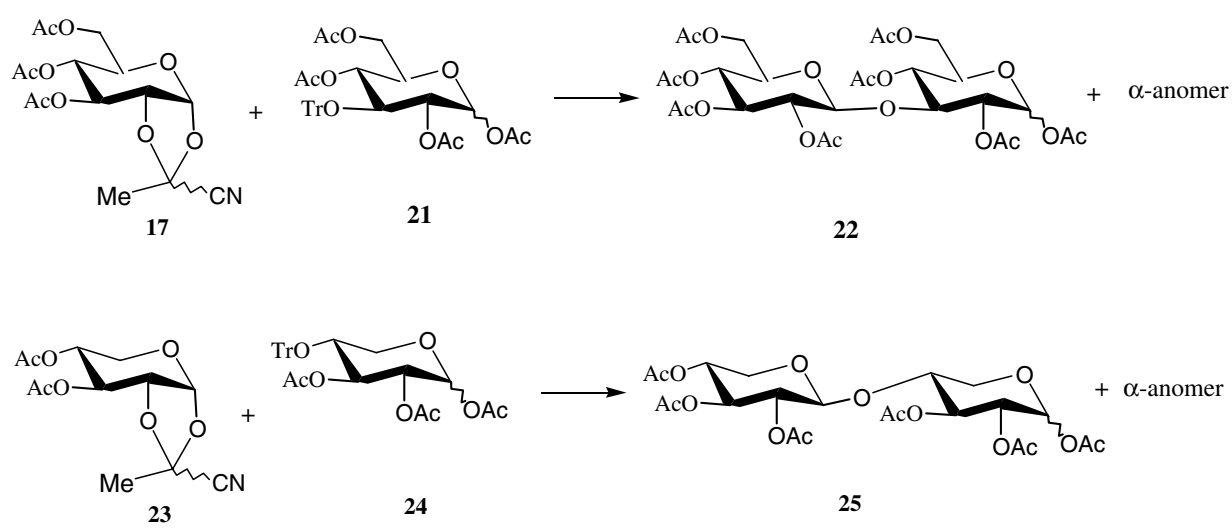

Scheme 1. Reaction of cyano-ethylidene analogue of sugar orthoester. 

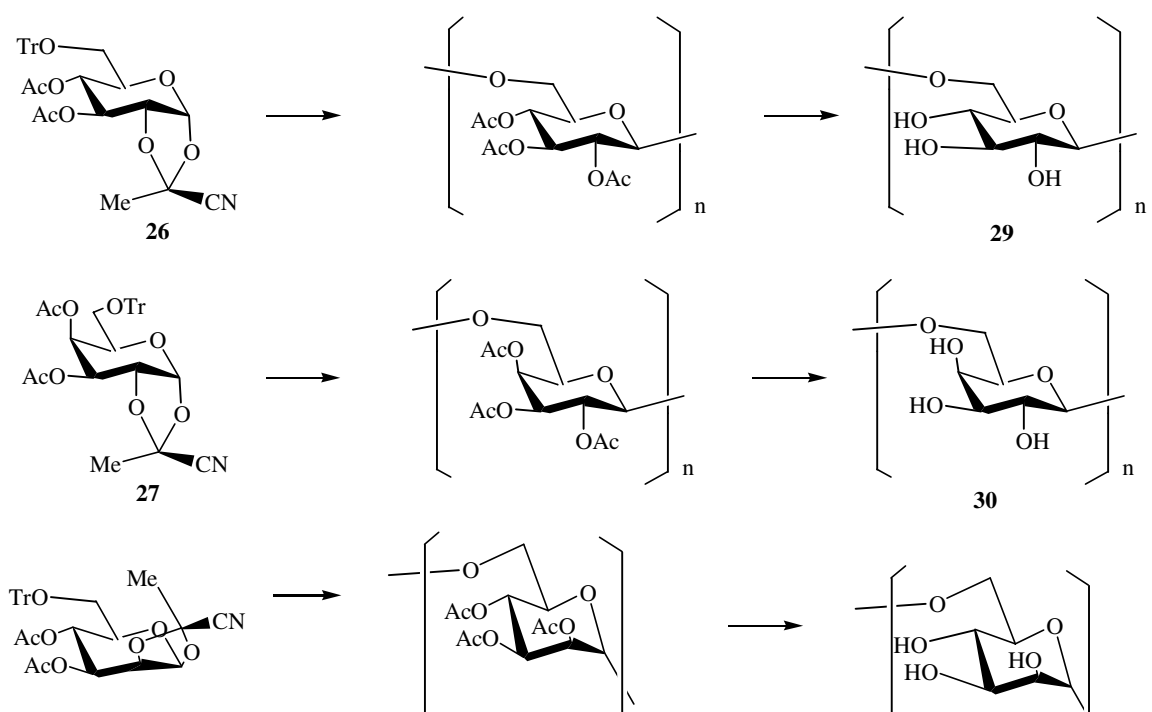

28
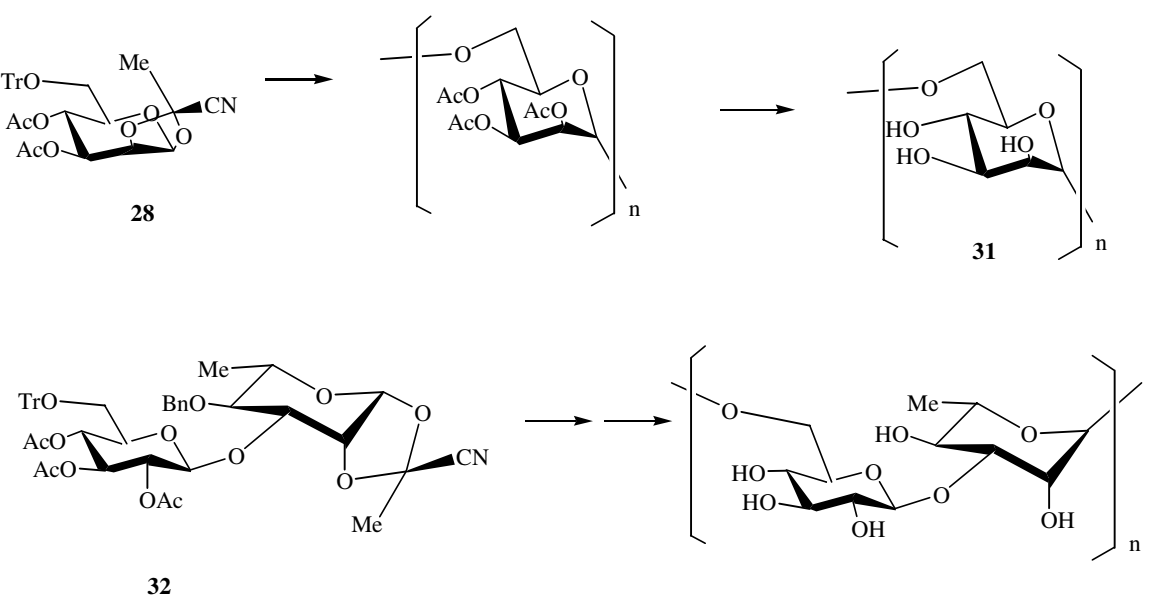

32

Scheme 2. Polymerization of mono- or disaccharide with cyano-ethylidene and trityl groups in the same molecule.

intra- and intermolecular rearrangement of the alkoxide. Now we will discuss recent studies on sugar-sugar orthoesters and their use in regioselective synthesis of 1,2-trans linked oligosaccharides, and abnormal synthesis of 1,2-cis linked oligosaccharides. Besides, we will also discuss the use of mannose orthoesters in the synthesis of $(1 \rightarrow 2)$ - $\alpha$-linked mannans.

\subsection{Preparation and identification of sugar-sugar orthoesters}

Sugar-sugar orthoesters are usually prepared $^{4 \mathrm{f}}$ by reaction of a glycosyl bromide donor with a glycosyl acceptor with only one free hydroxyl group in dichloromethane or 1,2-dichloroethane in the presence of an organic base such as 2,6-lutidine, or 2,4-lutidine, or 2,4,6-collidine. Silver nitrate, ${ }^{12 a}$ silver salicylate, ${ }^{12 b}$ silver triflate, ${ }^{4 \mathrm{~d}}$ silver imidazolate ${ }^{12 \mathrm{c}}$ can be used to promote the reaction. There are also some other reported methods, such as an approach through enhancement of the nucleophilicity of the acceptor hydroxyl group by trialkylstannylation, ${ }^{13 a}$ reaction of acetylglycosyl bromide with amide acetal in the presence of tetrabutylammonium bromide ${ }^{13 \mathrm{~b}}$ (Scheme 3 ) and reaction of a carbohydrate acceptor with $1,2-O$-vinylidene glycose $^{13 \mathrm{c}}$ (Scheme 4). Our experiences reveal that a system containing acetobromoglycose, sugar acceptor, dichloro- methane, silver triflate, and 2,4-lutidine is very convenient and highly efficient. ${ }^{13 \mathrm{~d}, \mathrm{e}}$

Identification of sugar-sugar orthoesters is readily carried out by chemical and spectrometric methods. The orthoesters are easily detected on TLC by their decomposition under weakly acidic conditions, and their NMR spectra usually show a signal for the methyl group of $\mathrm{CCH}_{3}$ at $\delta 1.6-1.7 \mathrm{ppm}$ and the orthoester $\mathrm{C}$ at $\delta 121-123 \mathrm{ppm}$.

\subsection{Catalysts for rearrangement of sugar-sugar orthoesters}

In early studies, ${ }^{2,3 b, 4 e, h}$ mercuric bromide has been used as the catalyst and nitromethane has been used as the solvent, and the rearrangement has been carried out under rather drastic conditions giving the product in fair yields. Later studies indicate that trimethylsilyl trifluoromethanesulfonate (TMSOTf), ${ }^{14 a-c}$ is a powerful catalyst under very mild conditions producing the product in high yields.

\subsection{Regio- and stereoselective synthesis of oligosaccha- rides via sugar-sugar orthoester intermediates}

The use of unprotected or partially protected sugars in glycosidic coupling is of particular interest since 


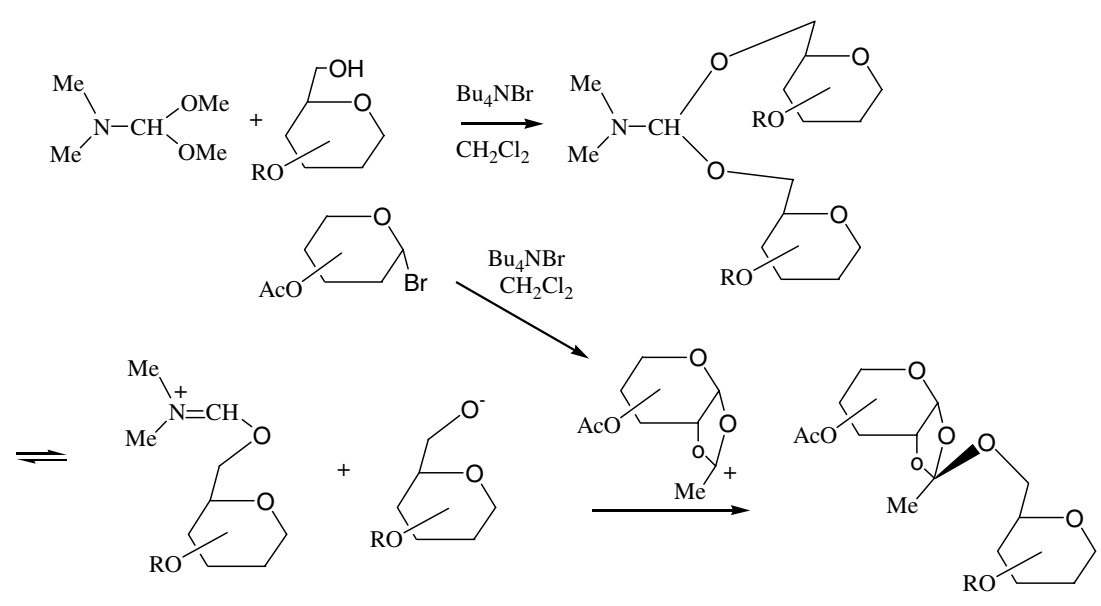

high overall yields

Scheme 3. Preparation of sugar-sugar orthoesters using amide acetal.
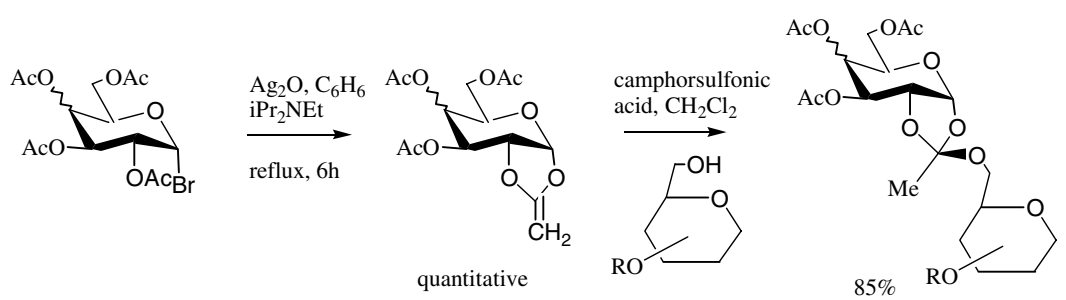

Scheme 4. Preparation of sugar-sugar orthoesters via 1,2-O-vinilydene glycose.

synthetic routes can be substantially simplified. In addition, chemistry on unprotected sugars represents a great challenge, as there are only tiny differences in coupling sugar hydroxyl groups, particularly secondary hydroxyl groups. There has been a review ${ }^{15 a}$ regarding the use of partially protected galactose acceptor in the synthesis of sialic acid-containing oligosaccharides. However, there have been very few reports dealing with the use of partially protected mannose acceptor, ${ }^{15 b-d}$ and there have been no reports dealing with the preparation of orthoesters using unprotected or partially protected glycosides as the glycosyl acceptors. Wang and Kong were the first to report ${ }^{16 a, b}$ that the preparation of sugarsugar orthoesters by coupling acetobromosugars with unprotected alkyl glycosides or partially protected sugar acceptors is achieved in high yields and excellent selectivity, and the rearrangement of either partially protected or fully protected orthoesters of diverse structure is achieved by the use of catalytic TMSOTf.

\subsubsection{Use of unprotected or partially protected glucosides} in regio- and stereoselective synthesis of sugar-sugar orthoesters. As shown in Scheme 5, coupling of acetobromoglucose 33 (1 equiv) with unprotected glucoside $\mathbf{3 4}$ (1 equiv) in DMF in the presence of AgOTf (1 equiv) and 2,4-lutidine (1.4 equiv) selectively affords the orthoester 35 , its in situ acetylation followed by rearrangement yielding disaccharide $\mathbf{3 6}$ in good yield. Also, reactions of $\mathbf{3 3}$ with 2,3-unprotected glucoside $\mathbf{3 7}$, with 3,4-unprotected glucoside $\mathbf{4 0}$, and with 4,6-unprotected glucoside $\mathbf{4 3}$ in dichloromethane regioselectively produce the 3-linked orthoesters $\mathbf{3 8}, \mathbf{4 1}$, and 6-linked orthoester $\mathbf{4 4}$ in high yields, respectively. Acylation of 38, 41, and 44 followed by rearrangement with catalytic amount of TMSOTf give $\mathbf{3 9}, \mathbf{4 2}$, and $\mathbf{4 5}$, in satisfactory yields $(\sim 80 \%)$, respectively. Similar results were obtained for couplings of acetobromogalactose $\mathbf{4 6}$ with the 4,6-unprotected glucoside $\mathbf{4 3}$ and 3,4-unprotected glucoside 40, giving selectively 6-linked orthoester $\mathbf{4 7}$ and 3-linked orthoester 49, respectively, in high yields, and consequent rearrangement and acetylation gave the 1,2-trans-linked disaccharides $\mathbf{4 8}$ and 50, respectively. The rearrangement selectivity was confirmed by the fact that the two paths, that is, acetylation of $\mathbf{4 9}$ followed by rearrangement, and rearrangement of $\mathbf{4 9}$ followed by acetylation give the same disaccharide $\mathbf{5 0}$.

With disaccharide donor such as acetobromomaltose 51 and 3,4-unprotected acceptor 40, the reaction also selectively yielded 3-linked sugar orthoester $\mathbf{5 2}$, and its rearrangement selectively gave $\beta-(1 \rightarrow 3)$-linked trisaccharide 53. It was also noted that coupling of disaccharide donor acetobromolactose $\mathbf{5 4}$ with 2,3-unprotected acceptor 37 under normal Koènigs-Knorr conditions, that is, with silver triflate as the promoter and dichloromethane as the solvent but without addition of lutidine, gave pentasaccharide $\mathbf{5 5}$ as the only product even though the ratio 

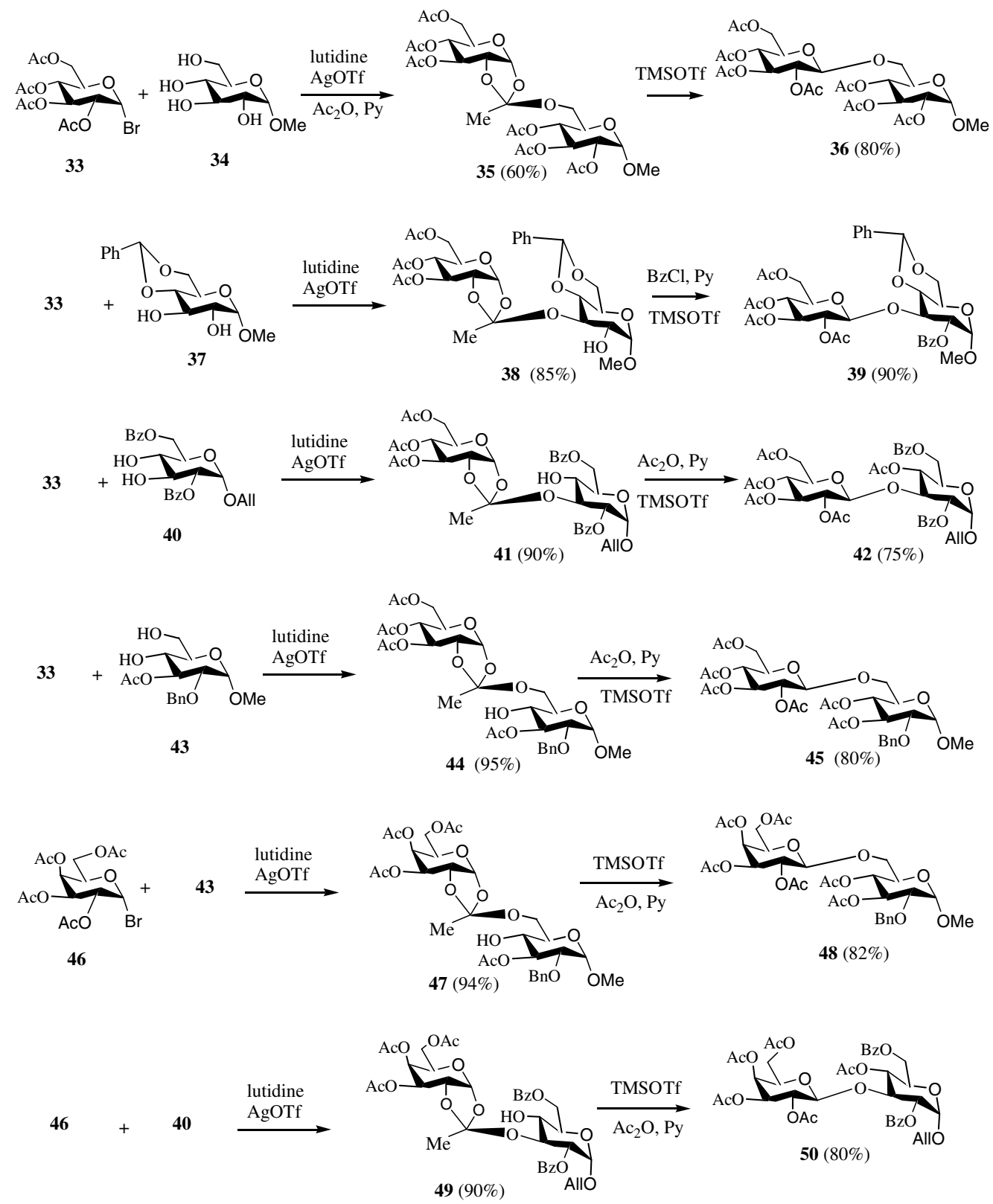

Scheme 5. Preparation and rearrangement of sugar-sugar orthoesters with unprotected or partially protected glucosides as the acceptors.

of donor/acceptor is one. This indicates that lutidine is necessary for orthoester formation since its basicity slows down the reaction rate enabling the reaction to take orthoester pathway with excellent selectivity. Without lutidine, the reaction goes very fast, and glycosidic bond is formed quickly even for the less active hydroxyl group leading to the loss of regioselectivity (see Scheme 6).

These results indicate that for partially protected glucoside acceptors, O-6 is the most active nucleophile, O-3 is the second in activity while O-2 and O-4 are relatively inert in the formation of sugar-sugar orthoesters. Besides, the regio- and stereoselectivities in the formation and rearrangement of sugar-sugar orthoesters are mainly dependent upon the acceptor rather than the donor since acetobromoglucose, acetobromogalactose, and acetylated biosyl bromide give the same or similar results.

3.3.2. Use of unprotected or partially protected mannosides in regio- and stereoselective synthesis of sugar-sugar orthoesters. Treatment of unprotected or partially protected mannosides with fully acetylated glucosyl- and mannosyl bromides under the same conditions as previously described also gives sugar-sugar orthoesters in high yields with high selectivity, while the rearrangement of the resultant orthoesters smoothly produces the required 1,2-trans-linked oligosaccharides. ${ }^{16 a, 17}$

Scheme 7 shows some typical examples with unprotected or partially protected mannosides as the acceptors. 

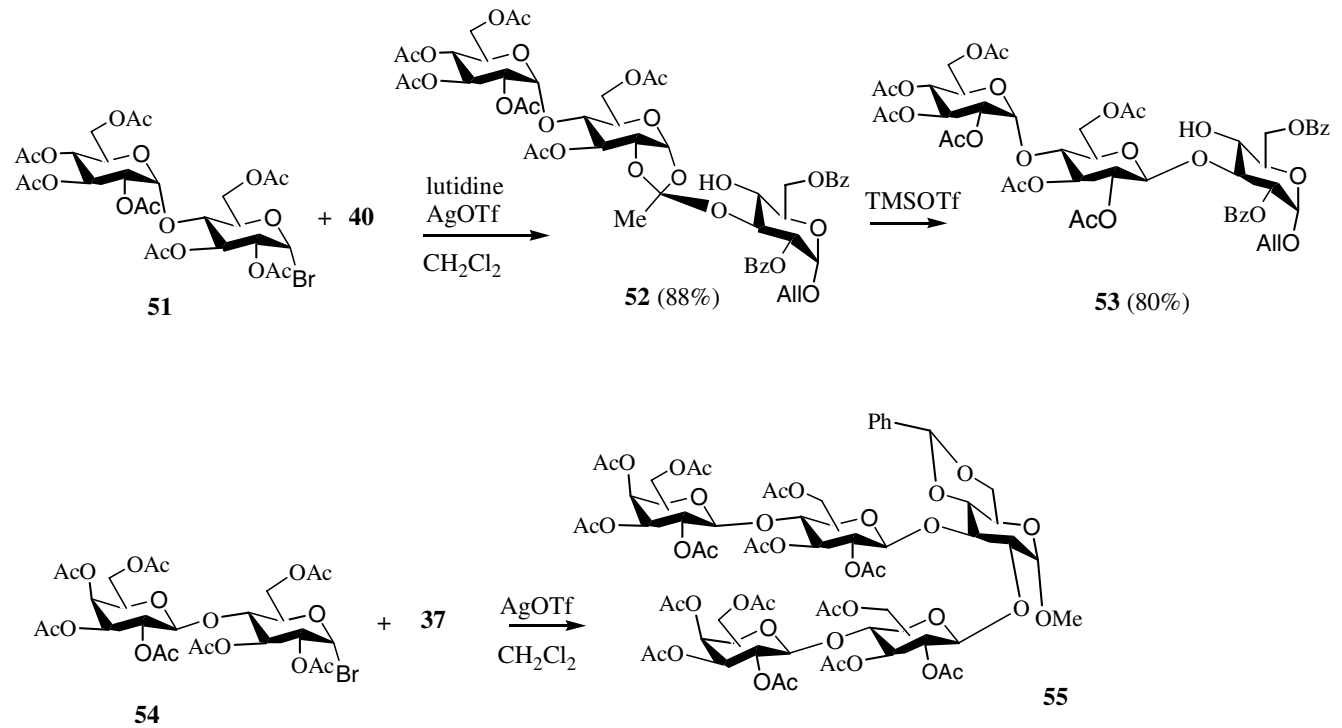

Scheme 6. Sugar-sugar orthoester formation with acetylated biosyl bromide donor.
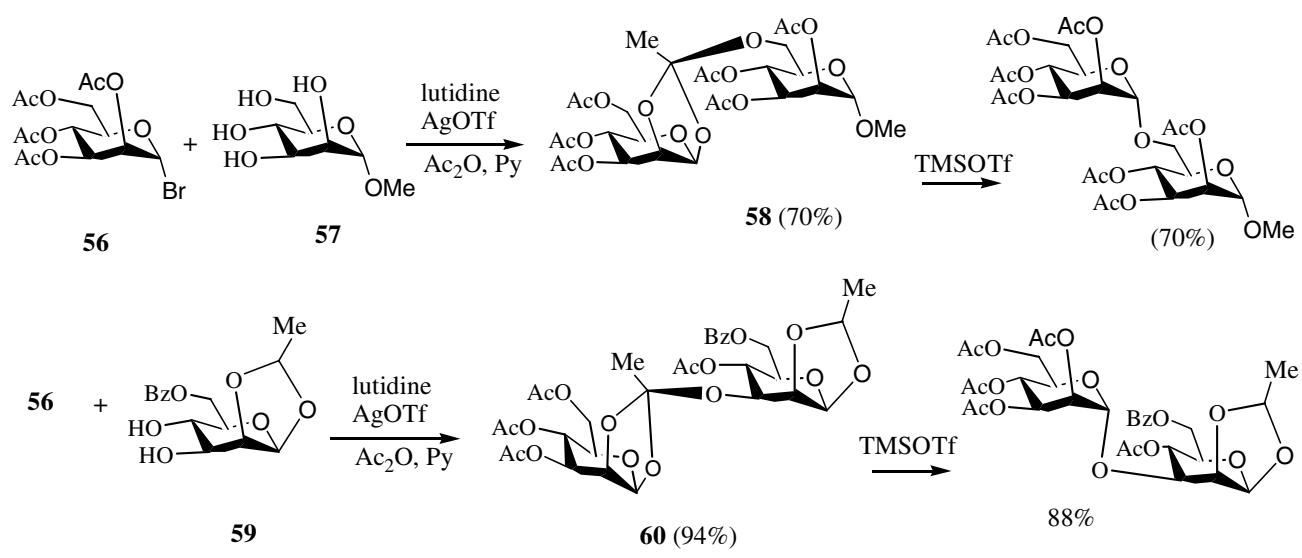

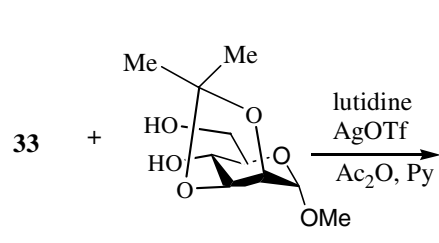

61

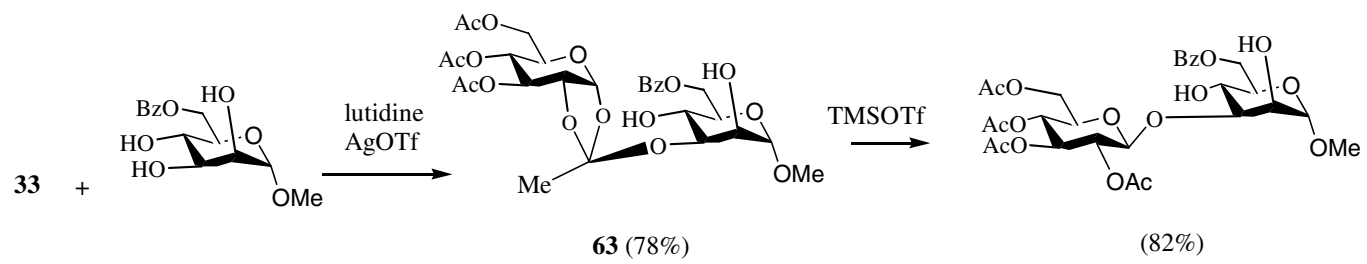

Scheme 7. Sugar-sugar orthoester formation with unprotected or partially protected mannose acceptors.

Coupling of acetobromomannose $\mathbf{5 6}$ (1 equiv) with unprotected mannoside 57 ( 1 equiv) in DMF under the orthoester formation conditions followed by acetylation gives 6-linked sugar-sugar orthoester $\mathbf{5 8}(70 \%),{ }^{16 a}$ while 
reaction of 56 with 3,4-unprotected mannose acceptor 59 and reaction of acetobromoglucose 33 with 4,6unprotected mannose acceptor $\mathbf{6 1}$ in dichloromethane selectively produce 3-linked and 6-linked sugarsugar orthoesters $60(94 \%)^{17}$ and $62(96 \%),{ }^{16 a}$ respectively. Rearrangement of the sugar-sugar orthoesters 58,60 , and 62 is carried out smoothly giving the corresponding $\alpha-(1 \rightarrow 6)-, \alpha-(1 \rightarrow 3)-$, and $\beta-(1 \rightarrow 6)$-linked disaccharides, respectively, in high yields. It is also interesting to note that the rearrangement of sugar orthoester $\mathbf{6 3}$ yields a partially protected $\beta-(1 \rightarrow 3)$-linked disaccharide indicating the high regioselectivity of the rearrangement.

In unprotected or partially protected mannose acceptors, the activity order of hydroxyl groups in the formation of sugar-sugar orthoesters is still $6-\mathrm{OH}>$ $3-\mathrm{OH}>2,4-\mathrm{OH}$, the same as that found in glucoside series.

3.3.3. Use of unprotected rhamnoside in regio- and stereoselective synthesis of sugar-sugar orthoesters. Reaction of unprotected rhamnoside with fully acetylated rhamnosyl bromide $\mathbf{6 4}$ in dichloromethane promoted by silver triflate in the presence of 2,4-lutidine selectively yielded 3-linked sugar-sugar orthoester $\mathbf{6 5}$ in high yield. Benzoylation of $\mathbf{6 5}$ followed by the rearrangement with TMSOTf as the catalyst produces $\alpha-(1 \rightarrow 3)$-linked disaccharide 66, and consequent selective deacetylation gave a disaccharide acceptor 67 with unprotected non-reducing rhamnose unit. Reiteration of coupling, benzoylation, and rearrangement transformed the disaccharide 67 to a trisaccharide ${ }^{18} \mathbf{6 8}$ as shown in Scheme 8.
3.3.4. Possible mechanism of sugar-sugar orthoester rearrangement. As described above for unprotected and partially protected sugar acceptors the orthoester formation-rearrangement-acylation or the orthoester formation-acylation-rearrangement afford the corresponding disaccharides in almost the same yields, the mechanism ${ }^{17}$ of the rearrangement is rationalized as shown in Scheme 9. When TMSOTf was added to the solution of 63 in dichloromethane, selective bond breaking at the orthoester linkage occurs giving closely packed ion pairs as shown in the form $\mathbf{A}$, and when this ion pairs reach an appropriate six-membered ring geometry as shown in the form $\mathbf{B}$, rearrangement occurs affording the required disaccharide. It is also supposed that the steric factor is the major factor responsible for the high regioselectivity in orthoester formation. This mechanism indicates that for sugar orthoesters consisting of gluco-, manno-, galacto- mono- or disaccharide as the donors and partially protected gluco-, manno-, and rhamnoside as the acceptors, their rearrangement mainly follows pathway (b) shown in Chart 1.

\subsection{Application of sugar-sugar orthoester formation- rearrangement in oligosaccharide synthesis}

3.4.1. Synthesis of phytoalexin elicitor hexaose. Phytoalexin elicitor hexaose can be synthesized by this method. ${ }^{13 \mathrm{~d}, 16 \mathrm{~b}}$ As outlined in Scheme 10, coupling of acetobromoglucose with a 4,6-unprotected disaccharide acceptor gives 6-linked sugar-sugar orthoester 69, its acetylation followed by rearrangement, then removal of ethylidene group, acetylation, 1-O-deacetylation, and
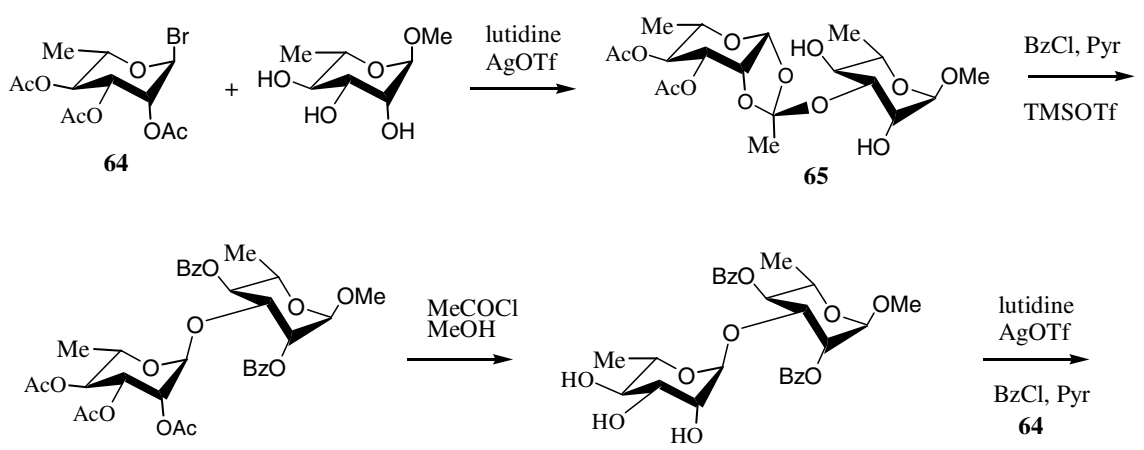

66

67

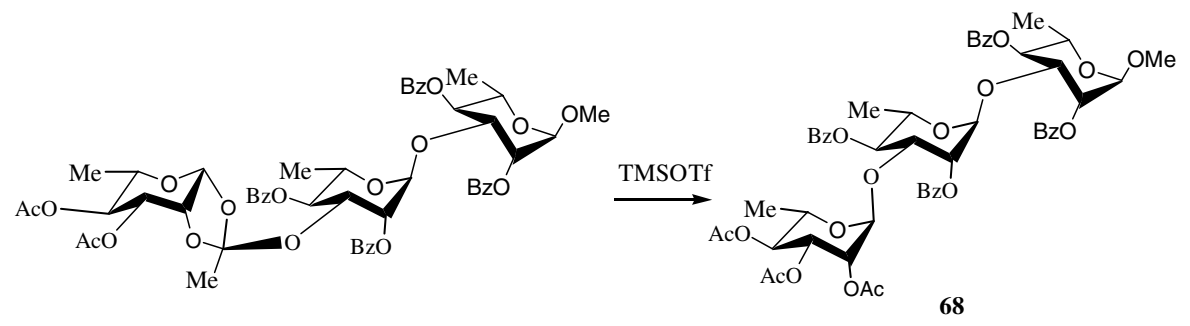

Scheme 8. Use of unprotected rhamnoside acceptor in regio- and stereoselective synthesis of sugar-sugar orthoesters. 


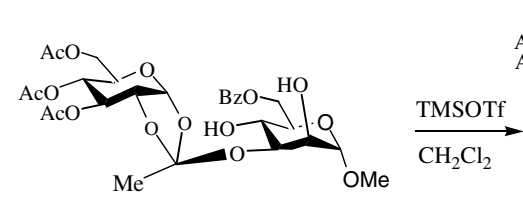

63

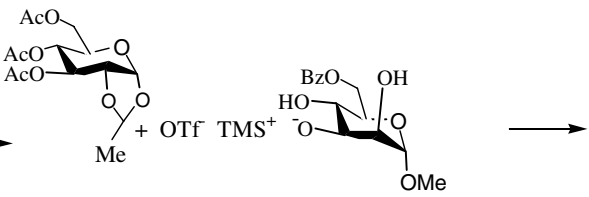

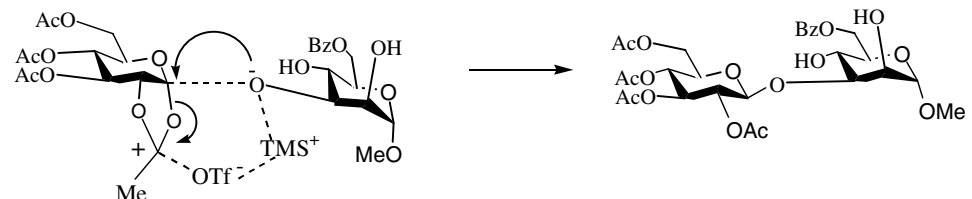

B

Scheme 9. Possible mechanism for sugar-sugar orthoester rearrangement.
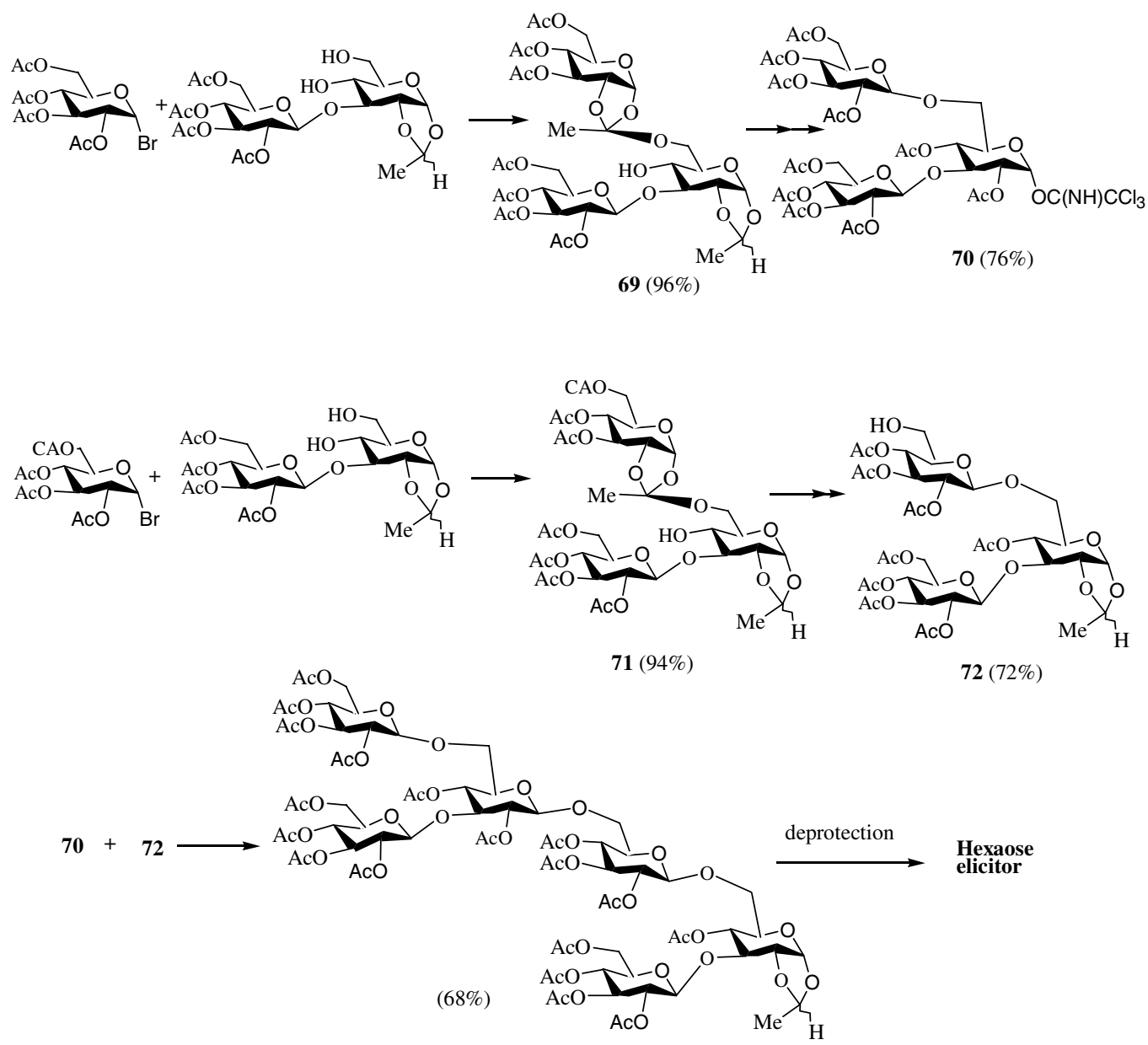

Scheme 10. Synthesis of phytoalexin elicitor hexaose by sugar-sugar orthoester formation-rearrangement.

trichloroacetimidate formation produced the trisaccharide donor 70. Meanwhile, coupling of 6-O-chloroacetylated (OCA) acetobromoglucose with the same 4,6unprotected disaccharide acceptor gave 6-linked sugar- sugar orthoester 71, its acetylation followed by rearrangement and 6-O-dechloroacetylation afforded the trisaccharide acceptor $\mathbf{7 2}$. Condensation of $\mathbf{7 1}$ with $\mathbf{7 2}$ followed by deprotection yielded the phytoalexin elicitor hexaose. 


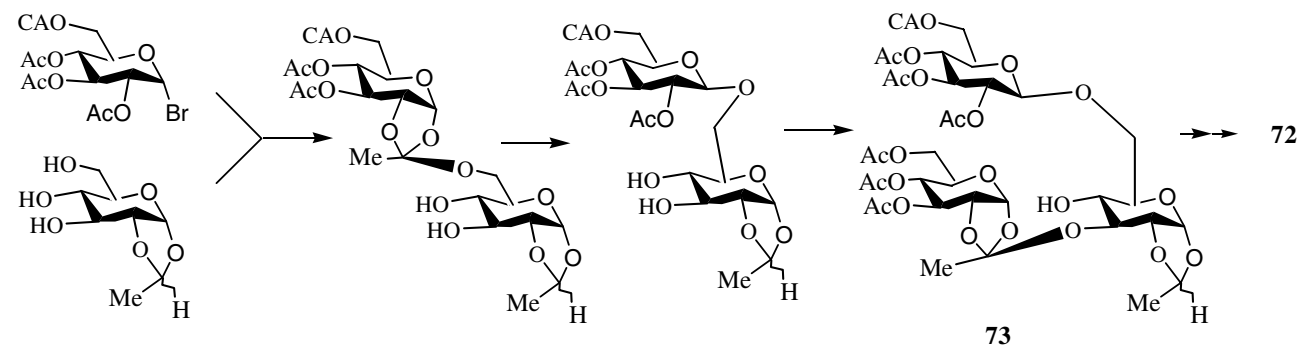

Scheme 11. Alternative preparation of trisaccharide acceptor.

Alternatively, the trisaccharide acceptor $\mathbf{7 2}$ was obtained by another way, ${ }^{19 a}$ that was, preparation of 6-linked sugar-sugar orthoester is carried out first, then rearrangement afforded the $\beta-(1 \rightarrow 6)$-linked disaccharide, which are used as a sugar acceptor to react with acetobromoglucose producing 3-linked sugar-sugar orthoester 73. Acetylation of $\mathbf{7 3}$ followed by rearrangement and 6-O-dechloroacetylation gave the same trisaccharide acceptor 72 (Scheme 11).

More simply, coupling of acetobromoglucose ( 2.5 equiv) with $1,2-O$-ethylidene-D-glucose (1 equiv) promoted by silver triflate ( 2.2 equiv) with dichloromethane as the solvent in the presence of 2,4-lutidine (2.4 equiv) at $\mathrm{rt}$ afforded the 3,6-linked diorthoester $\mathbf{7 4}$ as the sole product, and no 4,6- or 3,4-linked diorthoester isomers were detected. ${ }^{19 b}$ Acetylation of $\mathbf{7 4}$ followed by rearrangement, removal of ethylidene group, acetylation, 1-O-deacetylation, and trichloroacetimidate formation produced the trisaccharide donor $\mathbf{7 0}$ (Scheme 12). The trisaccharide acceptor $\mathbf{7 2}$ was synthesized through diorthoester in a similar way. ${ }^{19 b}$

3.4.2. Synthesis of $\alpha-(1 \rightarrow 6)$-linked manno- and $\beta-(1 \rightarrow 6)-$ linked gluco- and galacto-oligosaccharides. Based on the above described method, $\alpha-(1 \rightarrow 6)$-linked mannose oligosaccharides were effectively synthesized ${ }^{19 \mathrm{c}}$ as shown in Scheme 13. In these syntheses, sugar chain was extended by one sugar unit after each glycosylation, and preparation of sugar acceptors was carried out by methanolysis to selectively remove the acetyl group in the presence of benzoyl groups with methanolic hydrogen chloride obtained in situ by addition of $\mathrm{AcCl}$ to
$\mathrm{MeOH}(1: 49 \mathrm{v} / \mathrm{v})$ to selectively remove $O$-acetyl groups in the presence of $O$-benzoyl groups (a modification of a procedure proposed by Kochetkov et al. ${ }^{19 \mathrm{~d}}$

$\beta-(1 \rightarrow 6)$-linked glucose and galactose tetrasaccharides were synthesized similarly ${ }^{19 c}$ as shown in Scheme 14.

3.4.3. Synthesis of $\mathbf{1}, 1$-linked oligosaccharides. $1 \beta, 1 \beta$ Linked oligosaccharides were also synthesized via the sugar-sugar orthoester formation-rearrangement method. For example, coupling of acetobromomaltose with a sugar acceptor with C-1- $\beta$-free hydroxyl group afforded 1-linked orthoester $\mathbf{7 5}$, its rearrangement gave the required $1 \beta, 1 \beta$-linked oligosaccharide. Based on this method, asymmetric 1,1-linked oligosaccharide can be obtained. For instance, coupling of acetobromolactose with 1,2,3,4-tetra- $O$-acetyl- $\beta$-galactopyranose followed by rearrangement gave an asymmetric $1 \beta, 1 \beta$-linked oligosaccharide 76 as shown in Scheme 15. Of course, $1 \beta$-gluco-, $1 \alpha$-manno-linked oligosaccharide can also be synthesized in this way. ${ }^{19 \mathrm{e}}$

\subsection{Self-condensation of mannose 1,2-allyl orthoester for preparation of $(1 \rightarrow 2)$-linked mannose oligosaccharides}

It is generally considered that pathway (d) in Chart 1 is a side or undesired reaction ${ }^{5 e}$ as it usually produces a glycoside with C-2-free hydroxyl group in low yields. However, recent studies revealed that under appropriate conditions for mannose allyl orthoester, it can be the main reaction leading to $\alpha-(1 \rightarrow 2)$-linked mannose disaccharide in high yields. Thus, it can be considered as selfcondensation of mannose allyl orthoester.
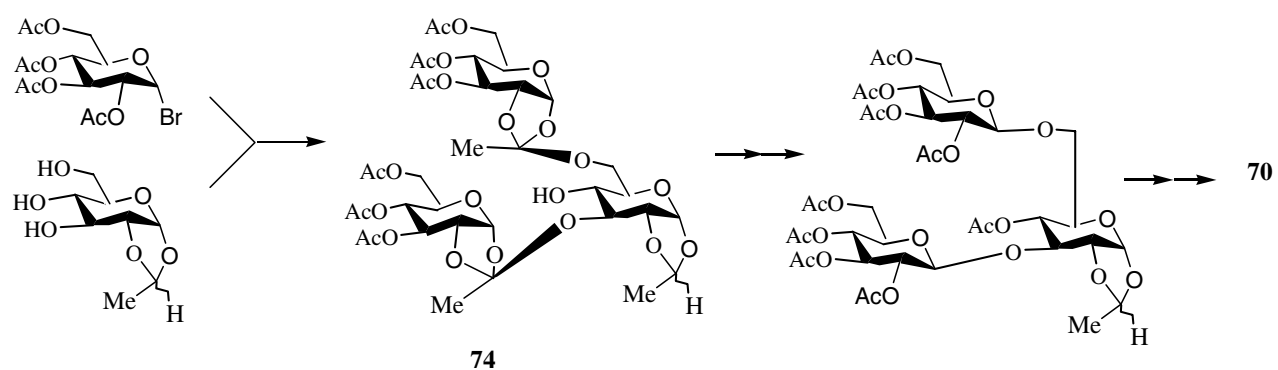

Scheme 12. Simplified synthesis of trisaccharide donor. 

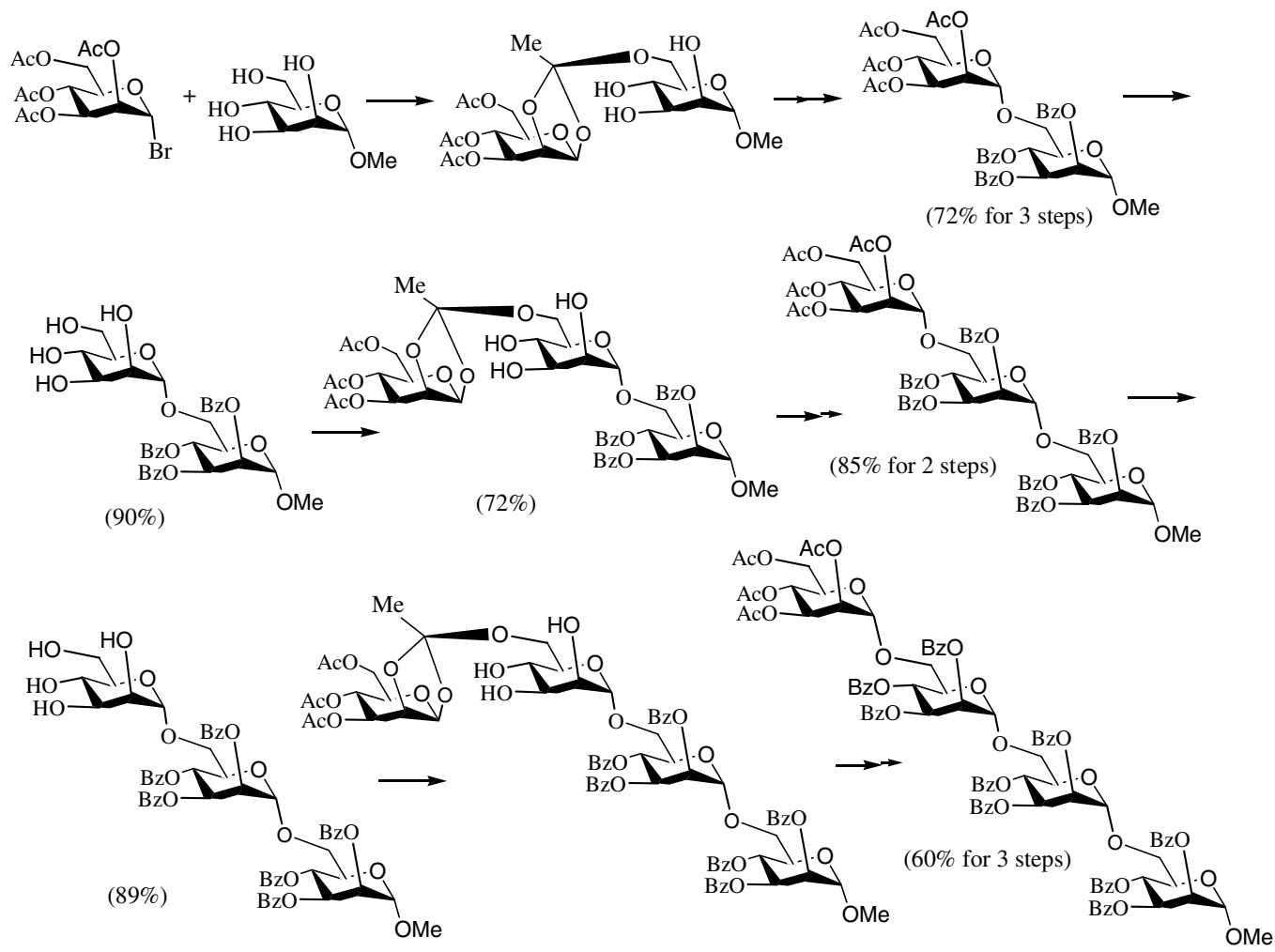

Scheme 13. Synthesis of $\alpha-(1 \rightarrow 6)$-linked mannose tetrasaccharide.
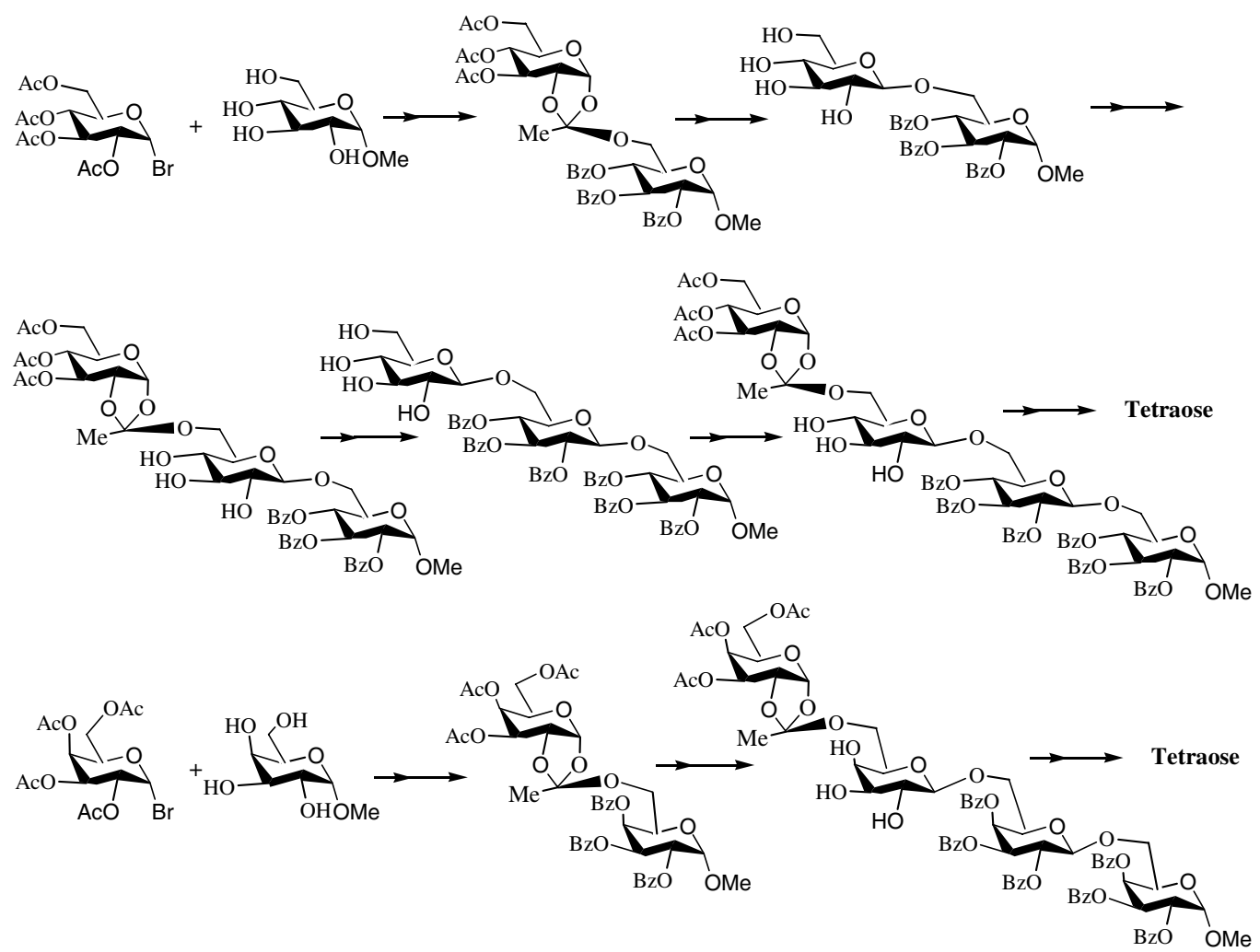

Scheme 14. Syntheses of $\beta-(1 \rightarrow 6)$-linked gluco- and galactose tetrasaccharides.

3.5.1. Mechanism of the self-condensation. Scheme 16 outlines the preparation and reaction mechanism ${ }^{20 a}$ of 3,4,6-tri- $O$-benzoyl- $\beta$-D-mannopyranose 1,2-allyl orthoester (77). Pathway (b) is the normal glycosylation of 

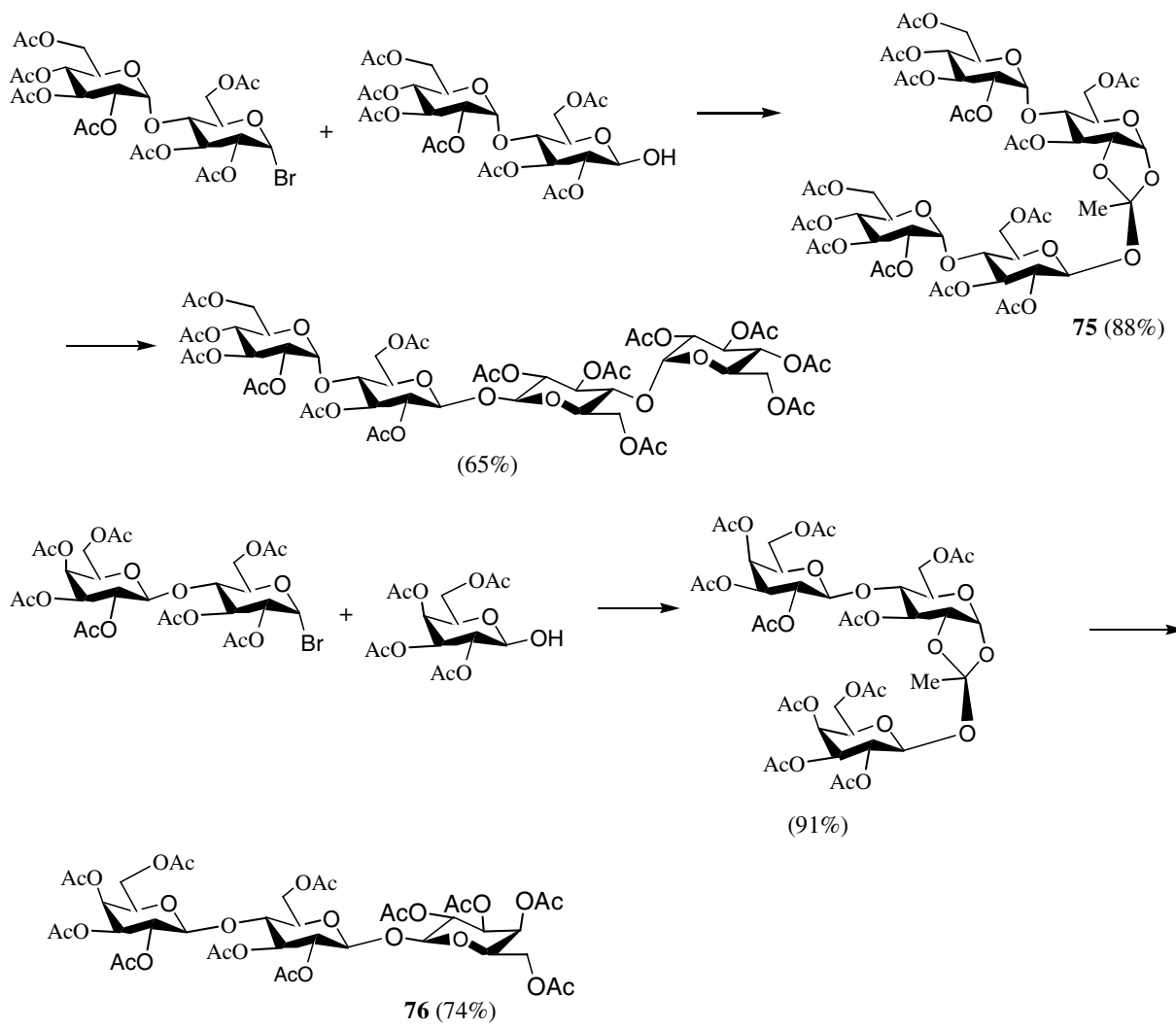

Scheme 15. 1,1-Linked oligosaccharide synthesis based on sugar orthoester method.

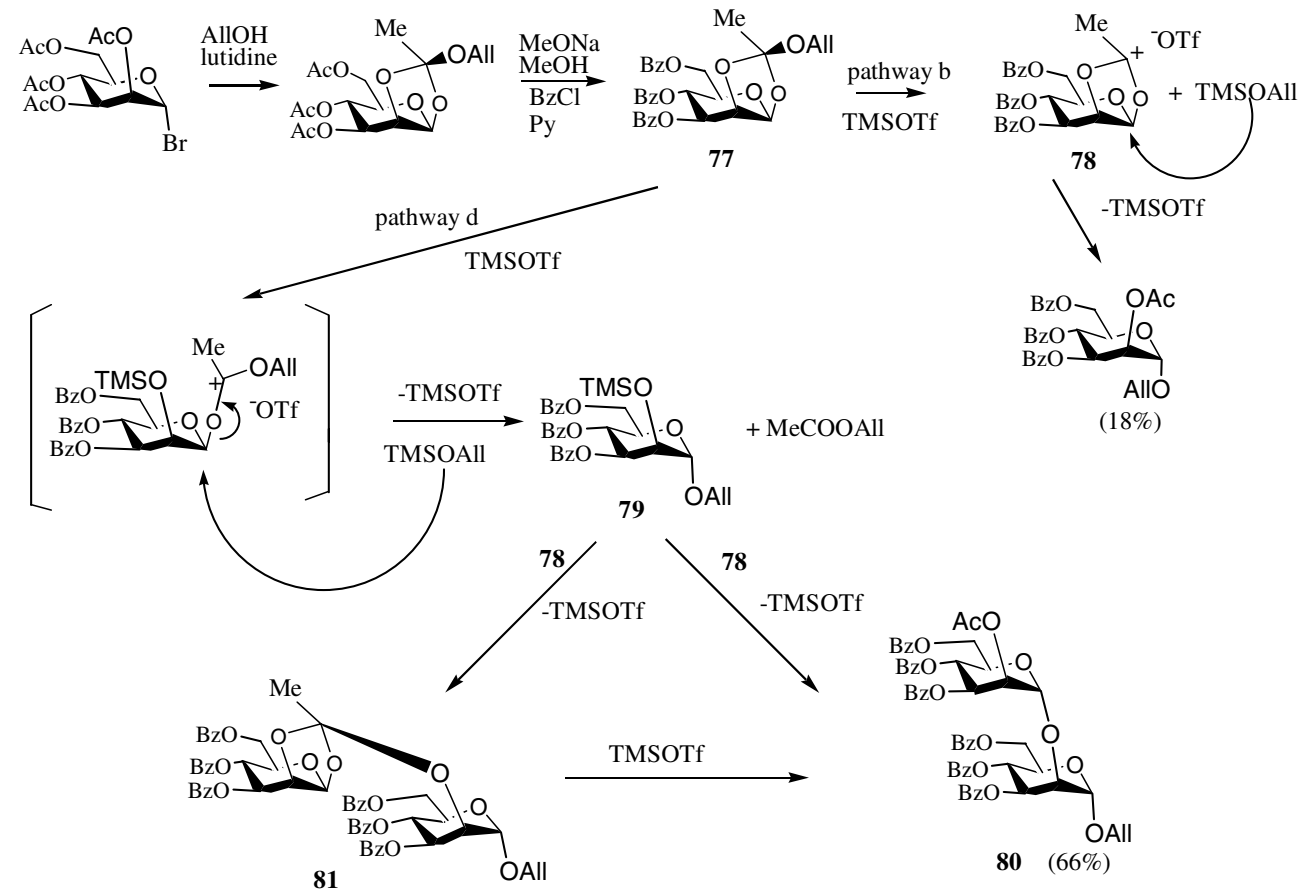

Scheme 16. Mechanism of self-condensation of mannose allyl orthoester.

the sugar orthoester $\mathbf{7 7}$ leading to the formation of allyl mannoside, while pathway (d) starts from the O-2$\mathrm{C}$ (orthoester) bond cleavage to produce 2-O-trimethyl- silylated allyl mannoside intermediate $\mathbf{7 9}$ that reacts with the active carbocation $\mathbf{7 8}$ obtained from pathway (b) to give an $\alpha-(1 \rightarrow 2)$-linked mannose disaccharide $\mathbf{8 0}$. 
3,4,6-Tribenzoylation in the orthoester 77 is essential since it enables pathway (d) to be the major reaction leading to the disaccharide 80 in high yield $(66 \%)$, while pathway (b) is the minor reaction leading to the allyl mannoside (18\%). Also, the tribenzoylation in $\mathbf{7 7}$ is very important as the resultant disaccharide contains at the O-2 of the nonreducing end sugar unit an acetyl group, which being different from the benzoyl groups can be selectively removed for further glycosylation. ${ }^{20 b-d}$

Further studies ${ }^{20 \mathrm{e}}$ revealed that in the condensation of orthoester 77, when the amount of catalyst TMSOTf is less than $5 \%$ of the orthoester 77 and the reaction time is relatively short $(2 \mathrm{~h})$, the product is a mixture of allyl mannoside (20\%), disaccharide $80(40 \%)$, and sugar-sugar orthoester $\mathbf{8 1}(40 \%$, Scheme 16). The latter was isolated in pure form and identified by NMR spectrometry. Rearrangement of $\mathbf{8 1}$ catalyzed by TMSOTf yielded the required disaccharide $\mathbf{8 0}$ in high yield. This indicates that sugar-sugar orthoester is the intermediate in the self-condensation of orthoester when the reaction rate is slowed down.

3.5.2. Self-condensation of rhamnose 1,2 -allyl orthoester for preparation of $(1 \rightarrow 2)$-linked rhamnose oligosaccharides. Self-condensation of rhamnose 1,2-allyl orthoester was also effectively achieved under similar conditions $^{20 \mathrm{a}}$ as indicated in Scheme 17. For the rhamnose orthoester $\mathbf{8 2}$, the reaction gave disaccharide $\mathbf{8 3}$ in higher yield $(72 \%$ for allyl orthoester, and $70 \%$ for methyl orthoester) and gave less allyl rhamnoside compared to the corresponding mannose orthoester.

3.5.3. Application of self-condensation of manno- and rhamnose orthoesters. The mannose disaccharide $\mathbf{8 0}$ and the rhamnose disaccharide $\mathbf{8 3}$, obtained from self-

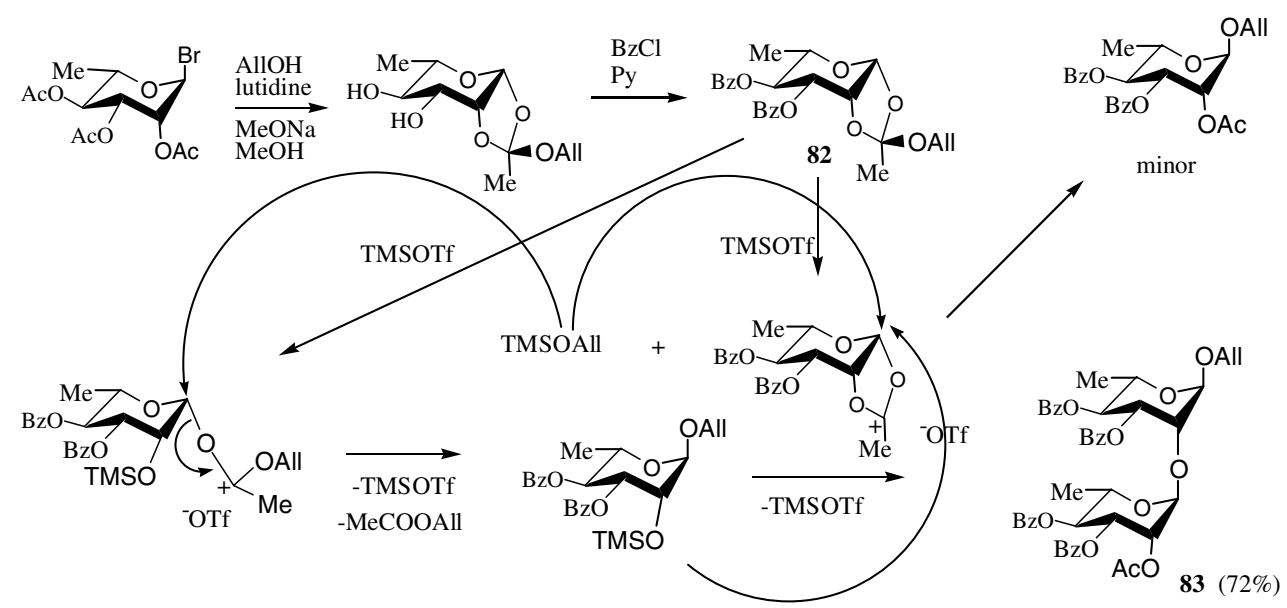

Scheme 17. Self-condensation of rhamnose orthoester.
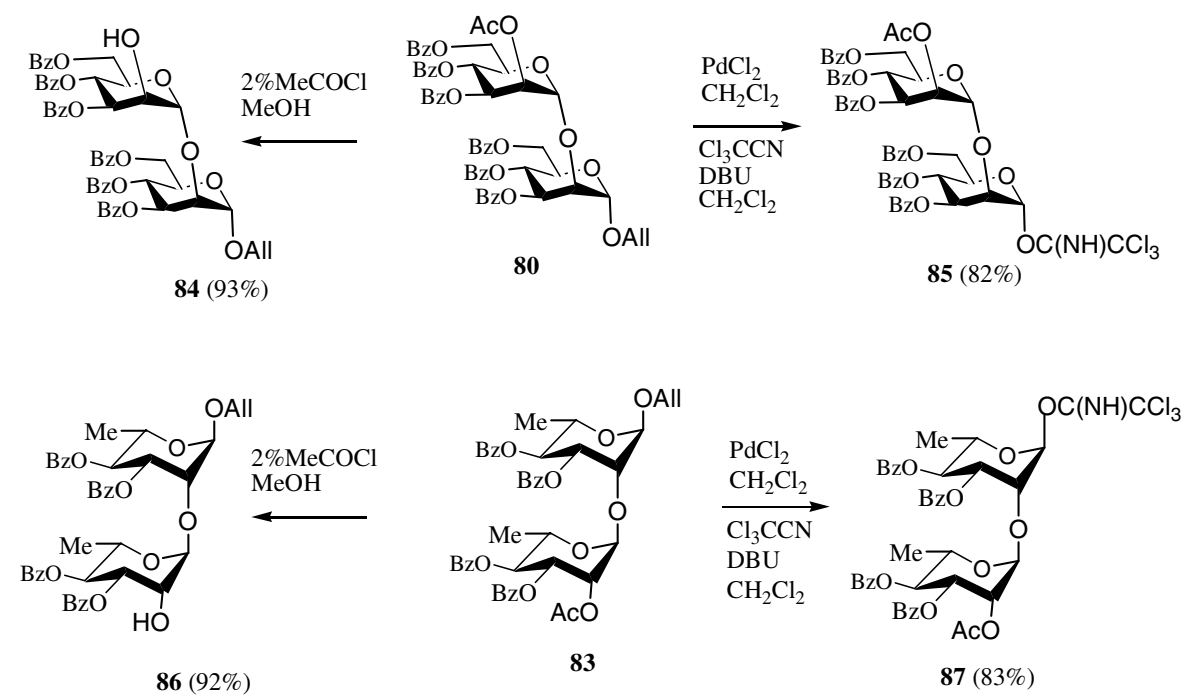

Scheme 18. Preparation of $\alpha-(1 \rightarrow 2)$-linked mannose and rhamnose disaccharide donor and acceptor. 
condensation of mannose orthoester and rhamnose analogue respectively, are very important building blocks for oligosaccharide syntheses since these disaccharides are readily transformed to either disaccharide donor or disaccharide acceptor as shown in Scheme 18. Deallylation followed by trichloroacetimidate formation of $\mathbf{8 0}$ and 83 afforded the disaccharide donors $\mathbf{8 5}$ and $\mathbf{8 7}$, respectively, in satisfactory yields. Meanwhile, selective
2'-O-deacetylation by methanolysis with methanolic hydrogen chloride obtained by mixing $2 \% \mathrm{MeCOCl}$ and $\mathrm{MeOH}$ gave the disaccharide acceptors 84 and $\mathbf{8 6}$ in high yields, respectively.

Many biologically important oligosaccharides were readily synthesized with these disaccharide building blocks. Some oligosaccharide structures are shown in Scheme 19, such as antigenic factor $4(\mathbf{8 8}),{ }^{20 \mathrm{c}}$ antigenic

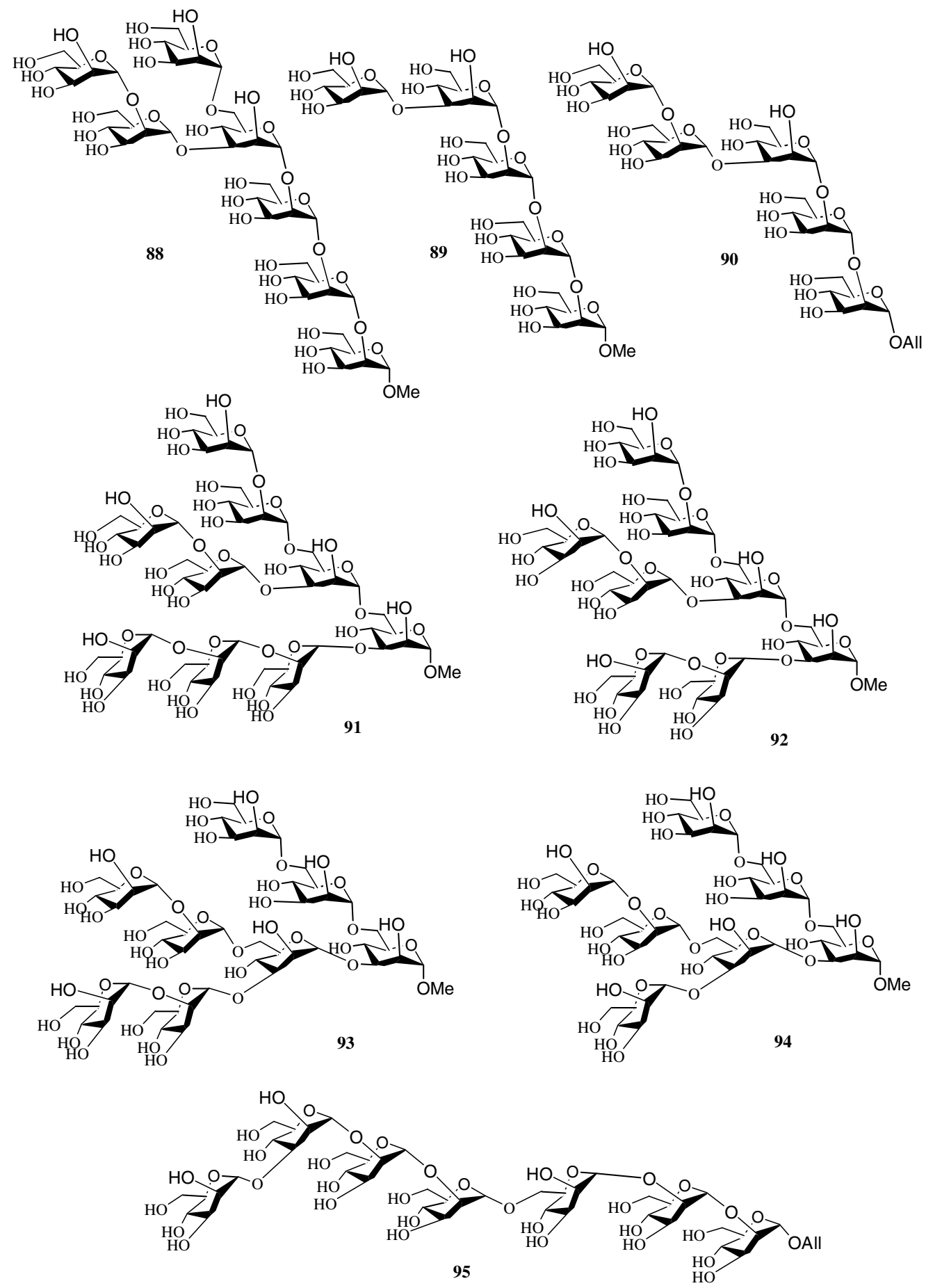

Scheme 19. Some mannose oligosaccharides synthesized by the new method. 

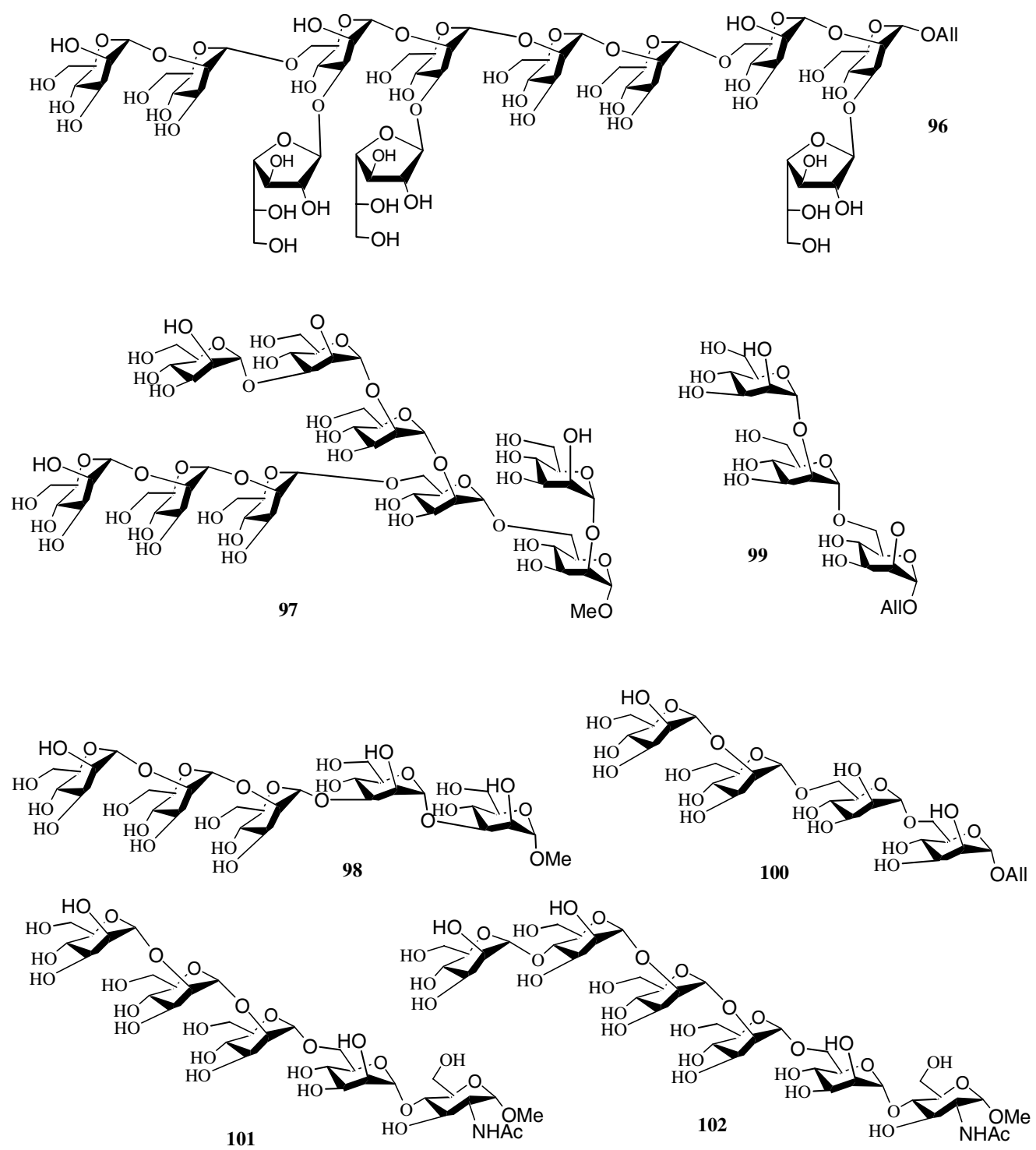

Scheme 19. (continued)

factor $34(\mathbf{8 9}),{ }^{20 \mathrm{~b}}$ antigenic factor $13(\mathbf{9 0}),{ }^{20 \mathrm{~b}}$ mannose nonasaccharide $(\mathbf{9 1})^{21 \mathrm{a}}$ of the $N$-glycan on the HIV glycoprotein gp 120, mannose octasaccharide $(\mathbf{9 2})^{21 b}$ of the $N$ glycan in human CD2, mannose octa- (93) and heptaose (94) analogues ${ }^{21 c}$ of the $N$-glycan mannans, and mannose heptasaccharides $(\mathbf{9 5})^{21 \mathrm{~d}}$ existing in Candida glabrata IFO 062 strain. Some important repeating units of microbial polysaccharides are also concisely synthesized, such as galactofuranosylated mannan repeating unit $(\mathbf{9 6})^{21 \mathrm{e}}$ of Trichophyton mentagrophytes IFO 5466 and Trichophyton rubrum IFO 5467, mannose nonasaccharide $(\mathbf{9 7})^{21 \mathrm{f}}$ in the exopolysaccharide of Cryphonectria parasitica, pentasaccharide repeating unit $(\mathbf{9 8})^{20 \mathrm{c}}$ of Escherichia coli $\mathrm{O} 9$ and Klebsiella $\mathrm{O} 3$, tri- (99) and tetrasaccharide $(\mathbf{1 0 0})^{20 \mathrm{~b}}$ repeating unit of Microsporum polysaccharide, and GPI anchor glycan oligosaccharides $(\mathbf{1 0 1}, \mathbf{1 0 2}) .^{21 \mathrm{~g}}$
3.5.4. A typical example of the use of O-2-(orthoester)C bond cleavage of mannose orthoester. It was reported ${ }^{21 \mathrm{~h}}$ that treatment of 2,6-di- $O$-acetyl-3,4-di- $O$-benzoyl- $\alpha$-Dmannopyranosyl trichloroacetimidate (103) with allyl alcohol (3-4 equiv) catalyzed by TMSOTf ( 0.3 equiv) in dichloromethane produced allyl 6-O-acetyl-3,4-di- $O$ benzoyl- $\alpha$-D-mannopyranoside (104) in an acceptable yield $(>30 \%)$. Obviously, compound $\mathbf{1 0 4}$ is obtained upon O-2-(orthoester)C bond breaking of the mannose allyl orthoester as shown in Scheme 20. Compound $\mathbf{1 0 4}$ is a valuable and key intermediate as it allows glycosylation at O-2 first to get the key disaccharide 106. This enabled the synthesis of a complex decasaccharide 107, the cell wall mannan of Candida kefyr IFO 0586, to be achieved $^{21 \mathrm{~h}}$ (Scheme 20) easily while it is very difficult to synthesize $\mathbf{1 0 7}$ by any other method. 

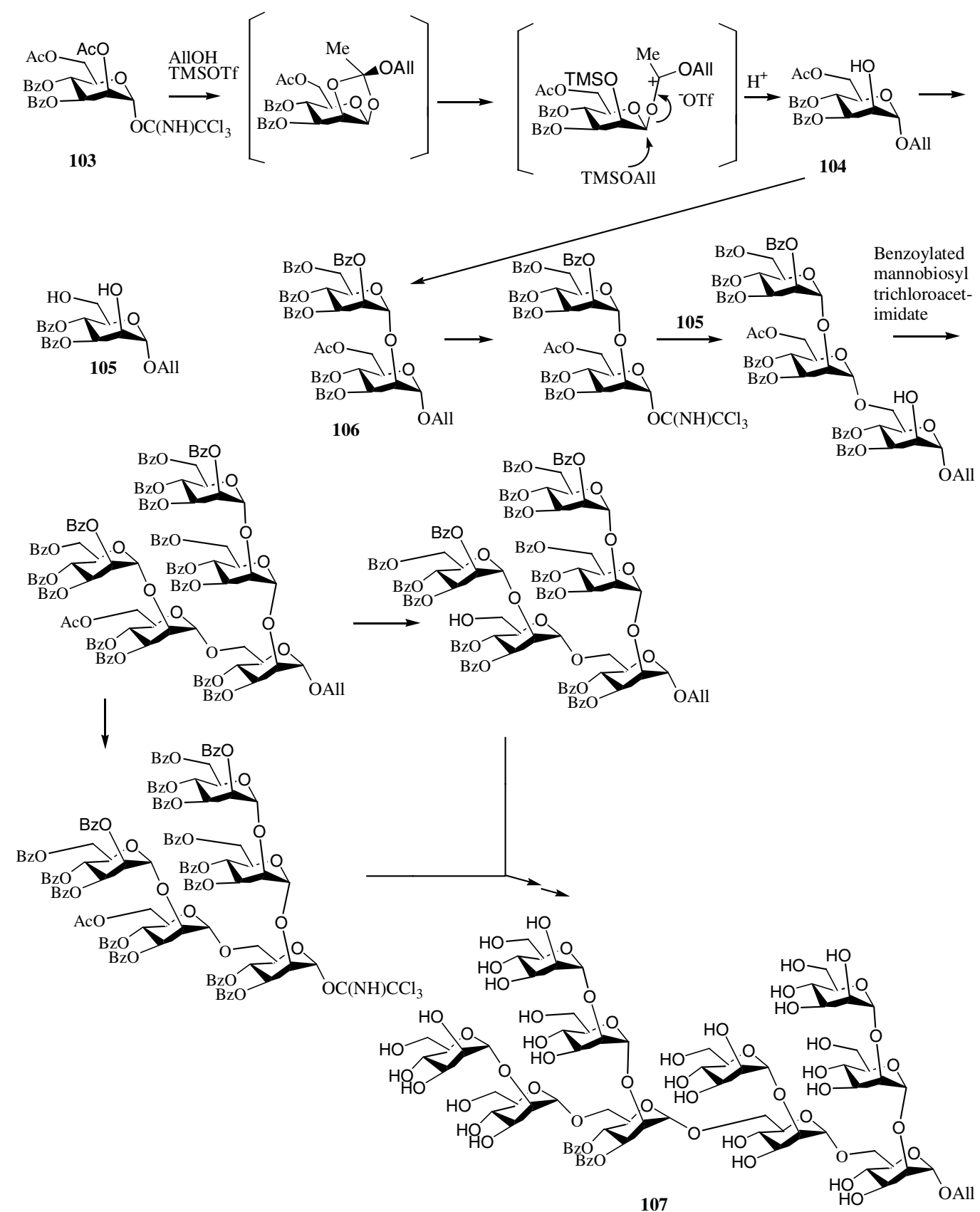

Scheme 20. Synthesis of cell wall mannan of Candida kefyr IFO 0586.

3.6. Regio- and stereoselective synthesis of oligosaccharides with sugar trichloroacetimidates as the donors and unprotected or partially protected glycosides as the acceptors via orthoester formation-rearrangement

As described in Section 3.4, regio- and stereoselective synthesis of oligosaccharides is achieved via orthoester formation-rearrangement with unprotected or partially protected glycosides as the acceptors. However, careful separation and purification of the orthoesters are needed for subsequent rearrangement and this makes the process complicated. For simplifying the process and for making large scale preparation possible, it is necessary to find a new way by which the orthoesters initially formed can rearrange to the required glycosides in situ. This one-pot, two-stage reaction will ensure the high regioselectivity of glycosylation as it comes from the high regioselectivity of orthoester formation. Meanwhile, elimination of steps of separation and purification of the orthoester will simplify the glycosylation substantially making large scale biosample preparations easy.

3.6.1. Sugar-sugar orthoester is usually the intermediate of glycosylation with sugar trichloroacetimidate as the donor. Glycosyl trichloroacetimidate donors are so far one of the most powerful intermediates in oligosaccharide 
synthesis. It was reported that in the coupling of 2,4,6-tri$O$-acetyl-3- $O$-methyl- $\alpha$-D-glucopyranosyl trichloroacetimidate with 1,2:5,6-diacetone glucofuranose, orthoester was the intermediate that converted to the corresponding glycoside by simply extending the reaction time. ${ }^{22 a}$ Recent studies revealed that orthoesters were also intermediates in the coupling reactions with mannosyl or glucosyl trichloroacetimidates as the donors and unprotected or partially protected glycosides as the acceptors, and the orthoesters were transformed to the corresponding glycosides in situ by addition of an extra catalyst or by extension of the reaction time. ${ }^{22 \mathrm{~b}}$ Thus, the glycosyl trichloroacetimidates become the ideal donors for the one-pot, two-stage glycosylations with unprotected or partially protected glycoside acceptors. For example, $\alpha-(1 \rightarrow 6)$-linked mannose dodecasaccharide was concisely synthesized as shown in Scheme 21 , and $\beta-(1 \rightarrow 6)$-linked glucose octasaccharide ${ }^{22 \mathrm{c}} \mathbf{1 1 2}$ was similarly obtained.

For the TMSOTf ( 0.08 equiv) catalyzed coupling of unprotected glycoside acceptor (1 equiv) with acetylated glycosyl trichloroacetimidate (1 equiv), DMF was used as the solvent, while for the couplings of partially protected glycoside acceptors dichloromethane was the solvent. It was noted that at the initial stage of the coupling reaction, orthoesters were formed (TLC); these were benzoylated and chromatographed to give pure

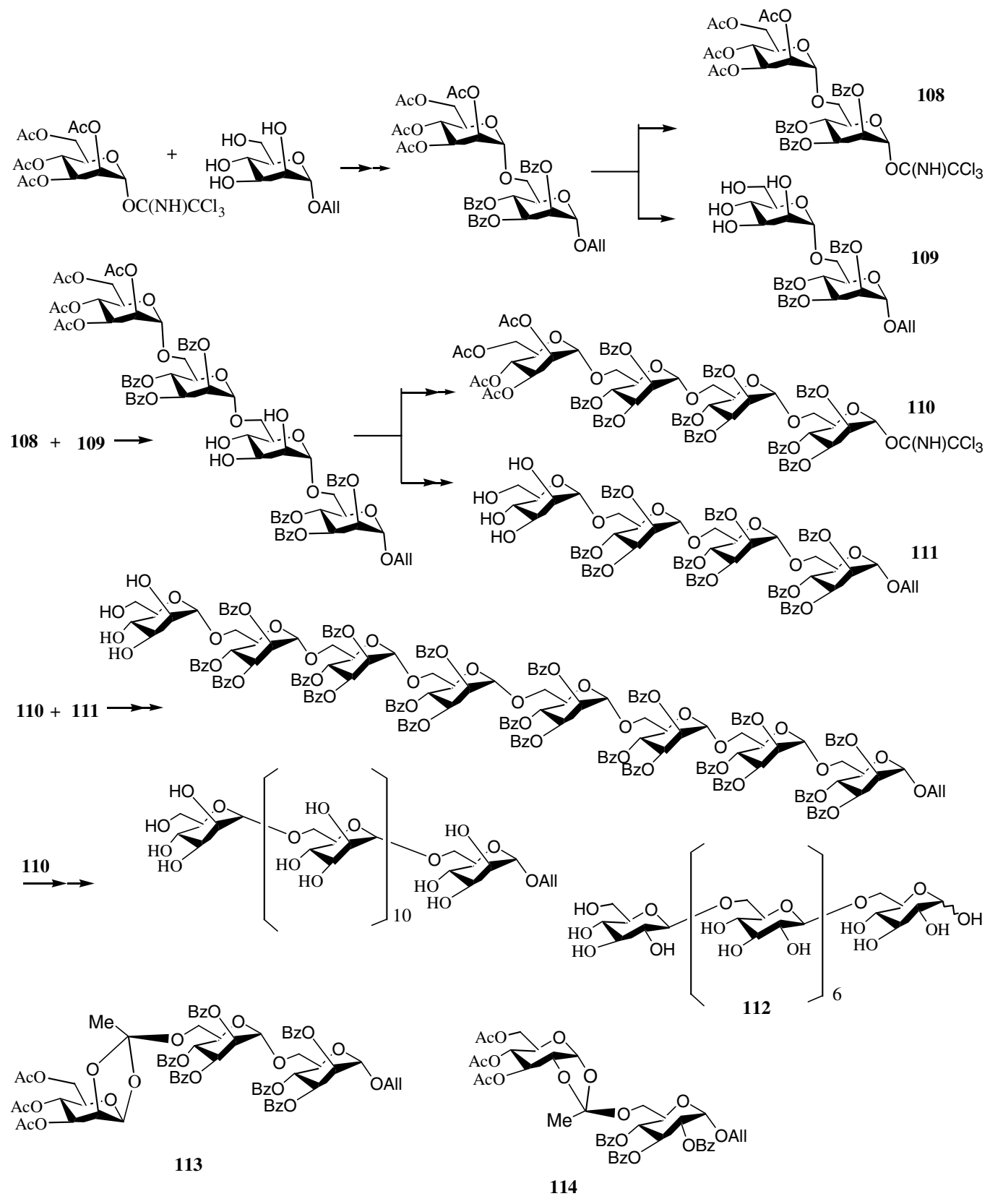

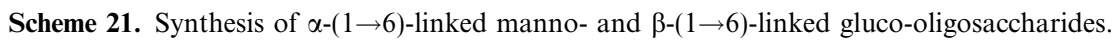




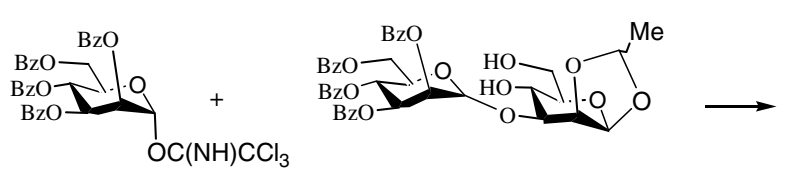

115

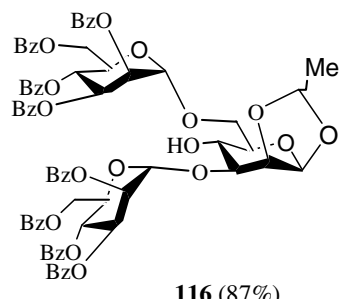

Scheme 22. Concise synthesis of 3,6-branched mannotriose.
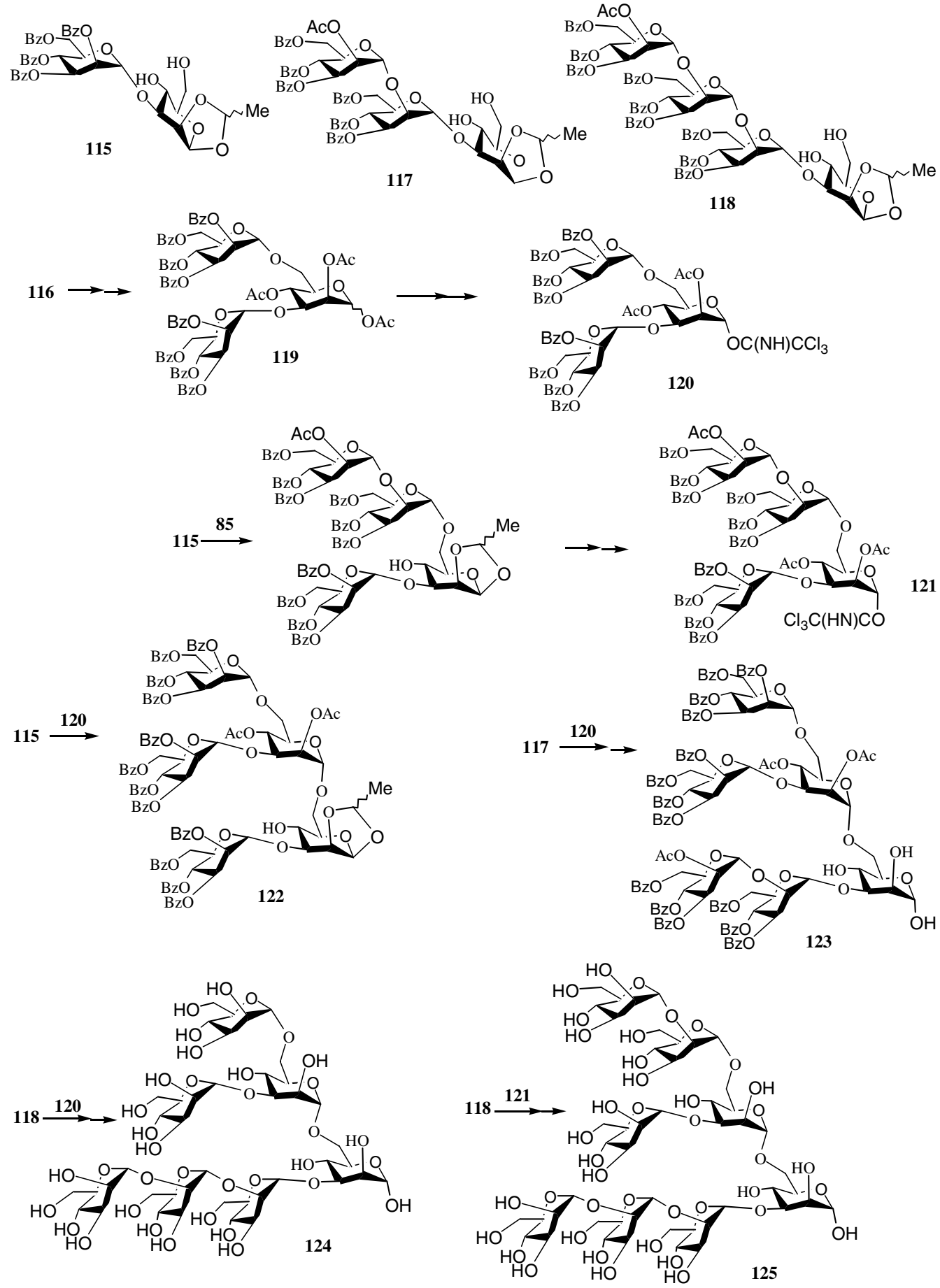

Scheme 23. Effective synthesis of multi-branched mannans in $N$-glycan. 
compounds 113 or 114 , indicating that orthoester is indeed the intermediate of the reaction.

\subsubsection{Application of glycosyl trichloroacetimidate donors} in regio- and stereoselective synthesis of biologically important oligosaccharides ${ }^{23 a}$

3.6.2.1. Synthesis of 3,6-branched mannotriose. 3,6Branched mannans exist in the core structure of $N$-glycan and syntheses of some 3,6-branched mannotriosides with unprotected mannoside acceptors are reported ${ }^{15 b-d}$ but the yields are not satisfactory. It was found that with partially protected mannose disaccharide $\mathbf{1 1 5}$ as the acceptor and benzoylated mannosyl trichloroacetimidate as the donor, the coupling reaction at low temperature yielded 3,6-branched mannotriose 116 in high yield $(87 \%)^{23 \mathrm{~b}}$ as shown in Scheme 22.

3.6.2.2. Synthesis of multi-branched mannans. With 4,6-unprotected mannose di- (115), tri- (117), and tetrasaccharide (118) as the acceptors and mannose di- (85), tri- (120), and tetrasaccharide trichloroacetimidate (121) as the donors, multi-branched mannopentaose (122), mannohexaose (123), mannoheptaose (124), and mannooctaose (125) existing in $N$-glycan were concisely and effectively synthesized in high yields ${ }^{23 b}$ as outlined in Scheme 23. For ensuring the high selectivity, all of the above coupling reactions were carried out at low temperature $\left(<0{ }^{\circ} \mathrm{C}\right)$ enabling the reaction to undertake orthoester formation pathway.

3.6.2.3. Synthesis of 2,6-branched mannan. 2,6Branched mannans existing in the cell wall polysaccharides of yeast such as Epidermophyton floccosum, $T$. mentagrophytes, Microsporum canis, Microsporum cookie, and Microsporum racemosum are also synthesized $^{23 c, 23 d}$ in a similar way as indicated in Scheme 24. Mannosylation of 2,6-unprotected mannose acceptor 105 with 6- $O$-acetyl-2,3,4-tri- $O$-benzoyl- $\alpha$-D-mannopyranosyl trichloroacetimidate selectively afforded $\alpha$ $(1 \rightarrow 6)$-linked disaccharide, and its mannosylation with fully benzoylated mannosyl trichloroacetimidate gave a 2,6-branched trisaccharide that was readily converted to trisaccharide acceptor $\mathbf{1 2 6}$ and trisaccharide donor 127. 2,6-Branched hexa- and nonasaccharides were easily obtained from $\mathbf{1 2 6}$ and $\mathbf{1 2 7}$.

3.6.2.4. Synthesis of rhamno-oligosaccharide. Some naturally occurring rhamnose oligosaccharides were concisely synthesized with unprotected rhamnoside as the acceptor and rhamnosyl trichloroacetimidate as the donor. The pentasaccharide repeating unit of LPS of Stenotrophomonas maltophilia serogroup O18 is a typical example. ${ }^{23 e}$ Coupling of fully acetylated rhamnosyl trichloroacetimidate with methyl rhamnoside selectively gave an $\alpha-(1 \rightarrow 3)$-linked disaccharide. Benzoylation fol-
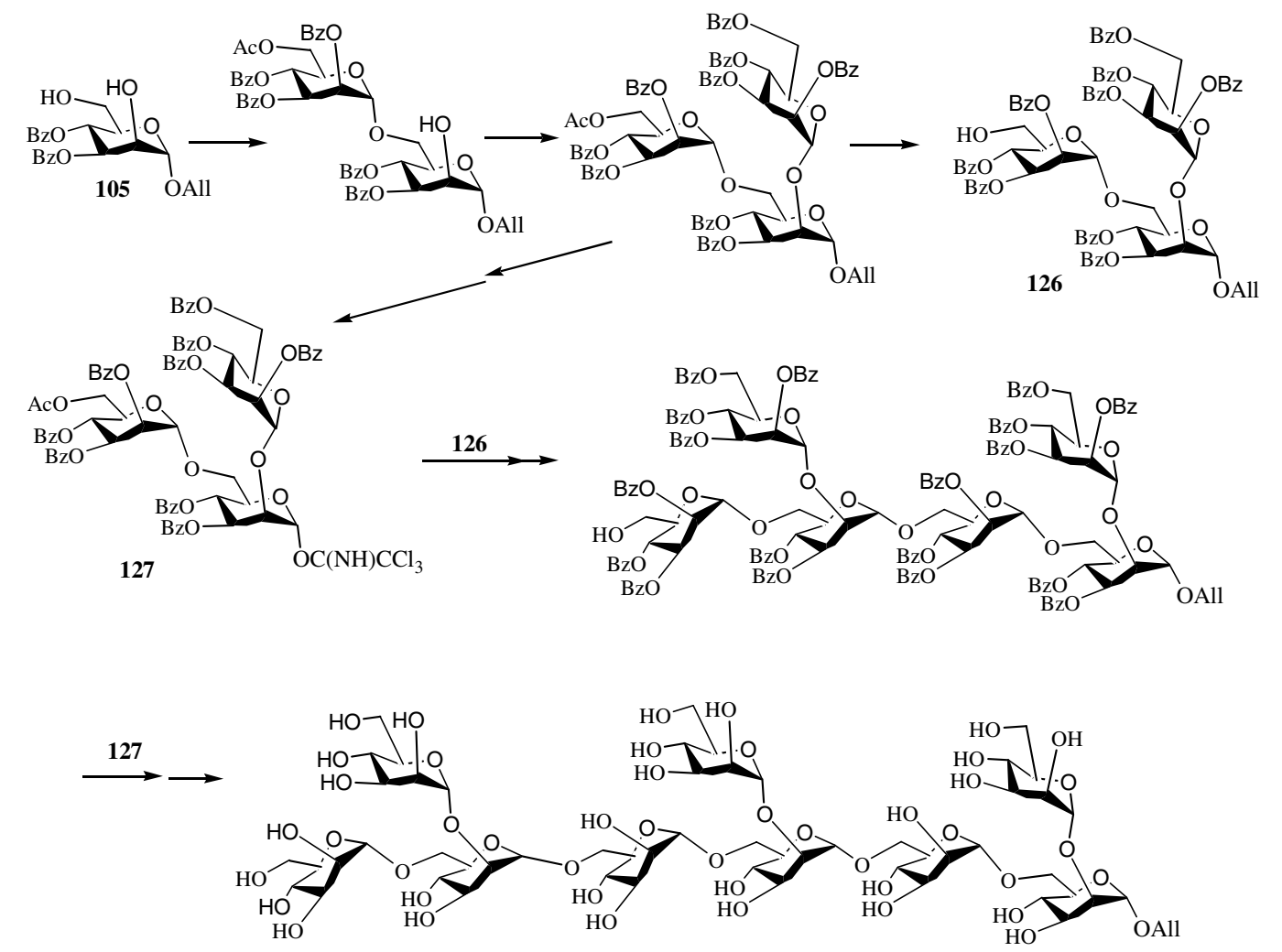

Scheme 24. Concise synthesis of 2,6-branched hexa- and nonasaccharide. 

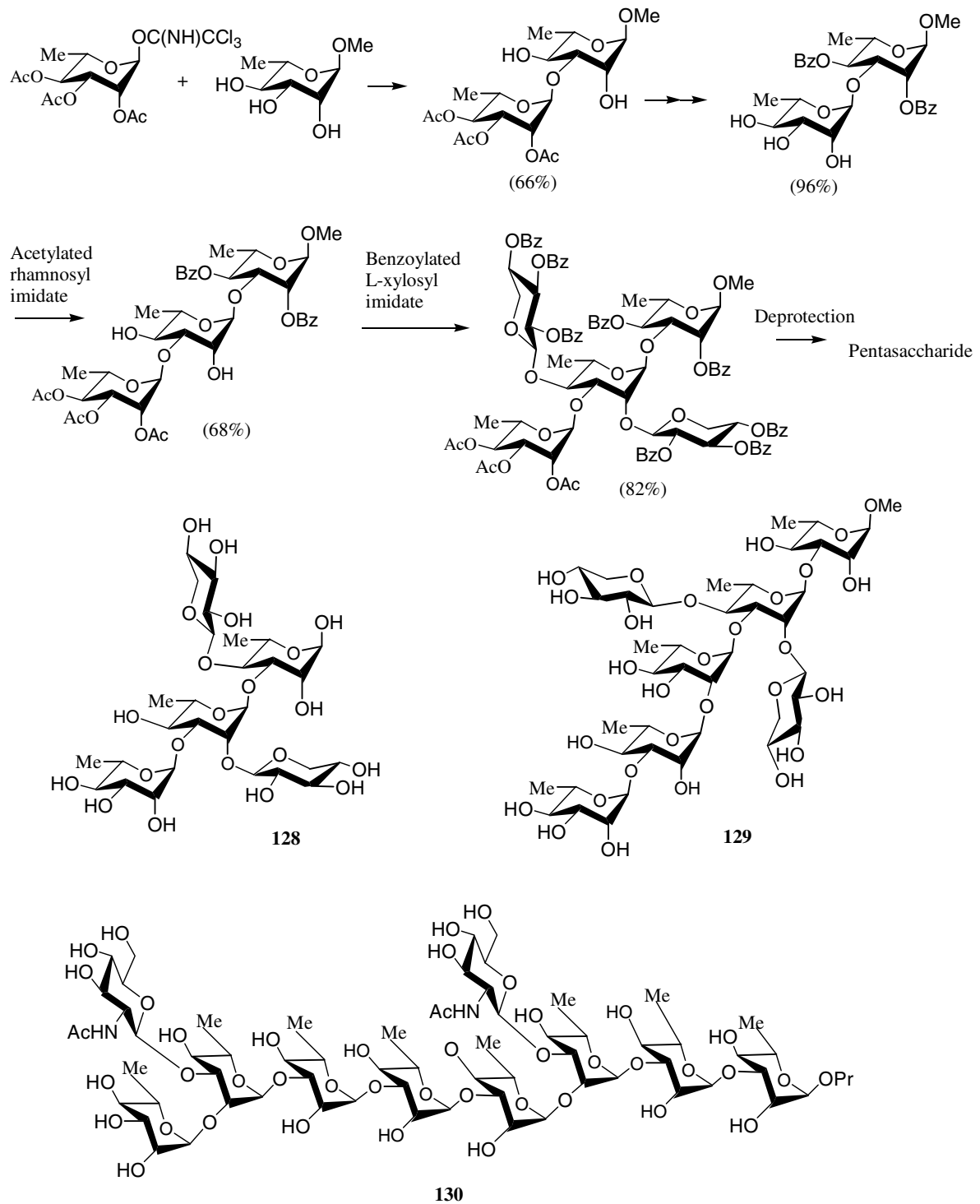

Scheme 25. Synthesis of a natural pentaose and some structures of rhamnose-containing oligosaccharides.

lowed by deacetylation produced a disaccharide acceptor with 2'-, 3'-, and 4'-free hydroxyl groups. Condensation of the disaccharide acceptor with fully acetylated rhamnosyl trichloroacetimidate gave an $\alpha-(1 \rightarrow 3)$-linked trisaccharide with $2^{\prime}$ - and $4^{\prime}$-free hydroxyl groups where the L-xylosyl side chain should be attached. Thus, the pentasaccharide was readily obtained by coupling the trisaccharide acceptor with benzoylated L-xylopyranosyl trichloroacetimidate followed by deprotection as shown in Scheme 25. The advantage of this method is clearly seen from this example.

Scheme 25 also shows the structures of another pentasaccharide repeating unit $(\mathbf{1 2 8})^{23 \mathrm{f}}$ of the O-chain polysaccharide of the lipopolysaccharide of Xanthomonas campestris pv. begoniae GSPB 525 and a heptasaccharide $(\mathbf{1 2 9})^{23 \mathrm{~g}}$ with $\mathrm{D}$-xylosyl side chains, the repeating unit of the O-chain lipopolysaccharide produced by $X$. campestris strain 642 , and they were also similarly syn- thesized. Synthesis of more complex rhamnose-containing oligosaccharides such as $\mathbf{1 3 0}^{\mathbf{2 3 h}}$ (Scheme 25) was completed in a similar way.

\subsubsection{Synthesis of the repeating unit of lentinan and} its analogues. A glucoheptaose consisting of $\beta-(1 \rightarrow 3)$ linked pentaose backbone with $\beta-(1 \rightarrow 6)$-linked glucosyl side chains, the repeating unit of lentinan, was synthesized as its allyl glycoside (131). ${ }^{23 i}$ A key step in the synthesis was the coupling of fully acetylated glucosyl trichloroacetimidate with partially protected $\beta-(1 \rightarrow 3)$ linked pentaose acceptor with two 4,6-unprotected glucose residues at the 2 nd and 4 th positions from the reducing end. It was found that the reaction selectivity was satisfactory giving $\beta$ - $(1 \rightarrow 6)$-linked heptaoside when the coupling was carried out at low temperature. With partially protected $\beta$ - $(1 \rightarrow 3)$-linked hexaoside acceptor with three 4,6-unprotected glucose residues at the 1st, 

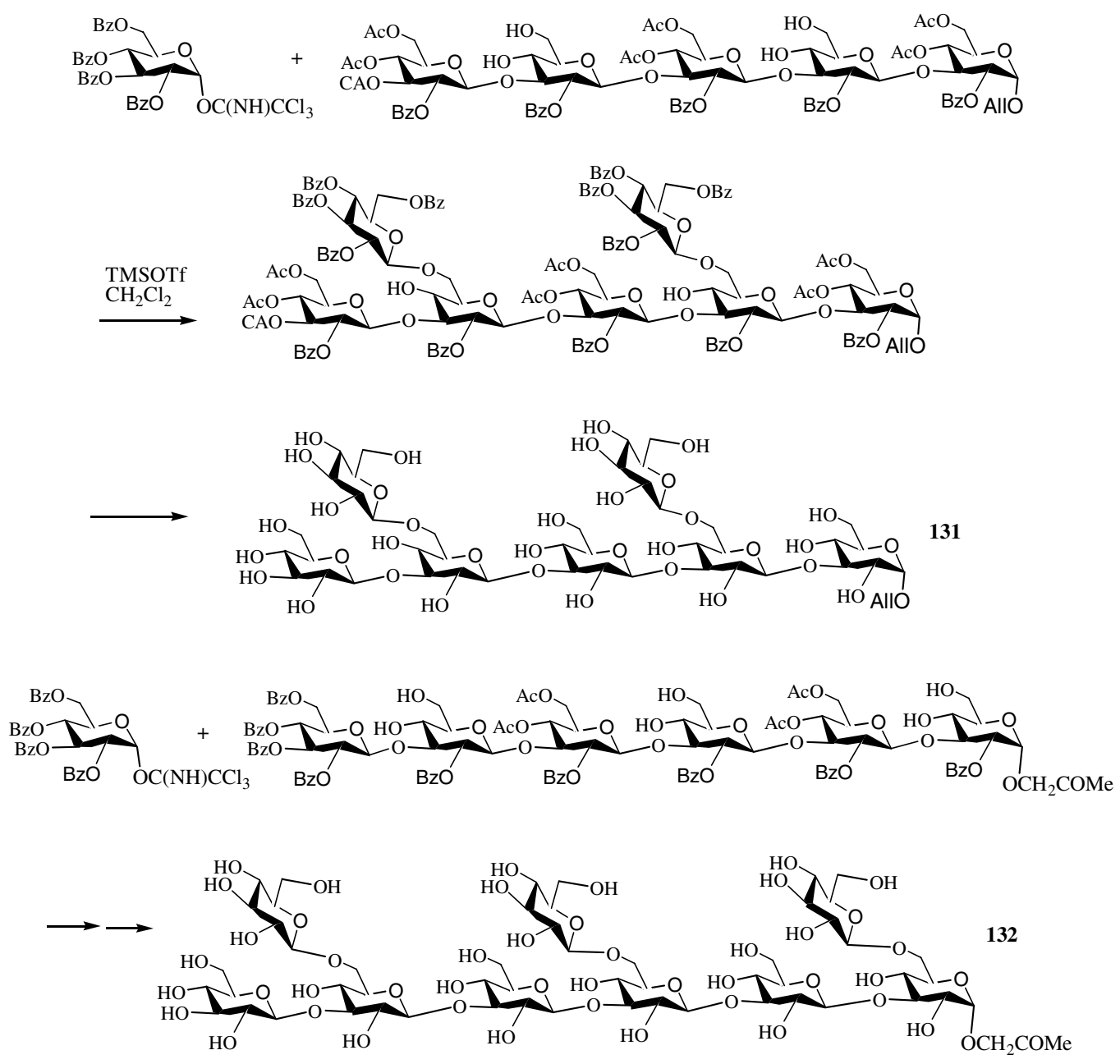

Scheme 26. Synthesis of the repeating unit of lentinan and its analogue.

$3 \mathrm{rd}$, and 5th positions from the reducing end, the selective coupling with fully acetylated glucosyl trichloroacetimidate gave a nonasaccharide $(\mathbf{1 3 2})^{23 \mathrm{j}}$ with three $\beta$ $(1 \rightarrow 6)$-linked side chains as shown in Scheme 26. Bioassay showed that in combination with the chemotherapeutic agent cyclophosphamide (CPA), the synthetic heptaoside at a dose of $0.5-1 \mathrm{mg} / \mathrm{kg}$ substantially increased the inhibition of $\mathrm{S}_{180}$ for CPA, but decreased the toxicity caused by CPA. ${ }^{23 \mathrm{k}}$

\subsubsection{Synthesis of phytoalexin elicitor glucohexaose} and its analogues. Highly effective and concise syntheses of the phytoalexin elicitor glucohexaose and its analogues were achieved ${ }^{231}$ with acylated glucosyl trichloroacetimidate as the donor and partially protected glucose as the acceptor in the presence of catalytic amount of TMSOTf. In the synthesis, selective coupling reactions with acylated glucosyl trichloroacetimidate donors and partially protected oligosaccharide acceptors were carried out producing the key trisacharides 133,135 , and hexasaccharide 137 as shown in Scheme 27. Owing to the use of partially protected glucosyl acceptors, the synthetic process was sub- stantially simplified enabling the production of the elicitor in hundreds grams scale in laboratory. ${ }^{23 \mathrm{~m}}$ At a concentration of 5-10 $\mathrm{mg} / \mathrm{L}$, the resultant elicitor hexaose is used to treat growing orange trees and harvested oranges, giving very encouraging results, comparable with those obtained using commercial pesticides at a concentration of $1400 \mathrm{mg} / \mathrm{L}$ (Topsin-M) for growing trees and $900 \mathrm{mg} / \mathrm{L}$ (Tecto) for harvested oranges, respectively. ${ }^{23 \mathrm{~m}}$ The elicitor glucoheptaose and its lauryl glycoside were synthesized in a similar way. ${ }^{23 n}$

3.6.3. Sugar-sugar orthoester is not always convertible to the desired oligosaccharide. Sugar-sugar orthoesters, formed as the intermediates in the coupling reactions with glycosyl trichloroacetimidates as the donors in the presence of catalytic amounts of TMSOTf, are detectable in many cases and separable in some cases. In most cases, the orthoesters are easily converted to the desired oligosaccharides in the presence of catalytic amounts of TMSOTf.

However, it was reported ${ }^{24 a}$ that in the synthesis of the octasaccharide repeating unit of the O-deacetylated 


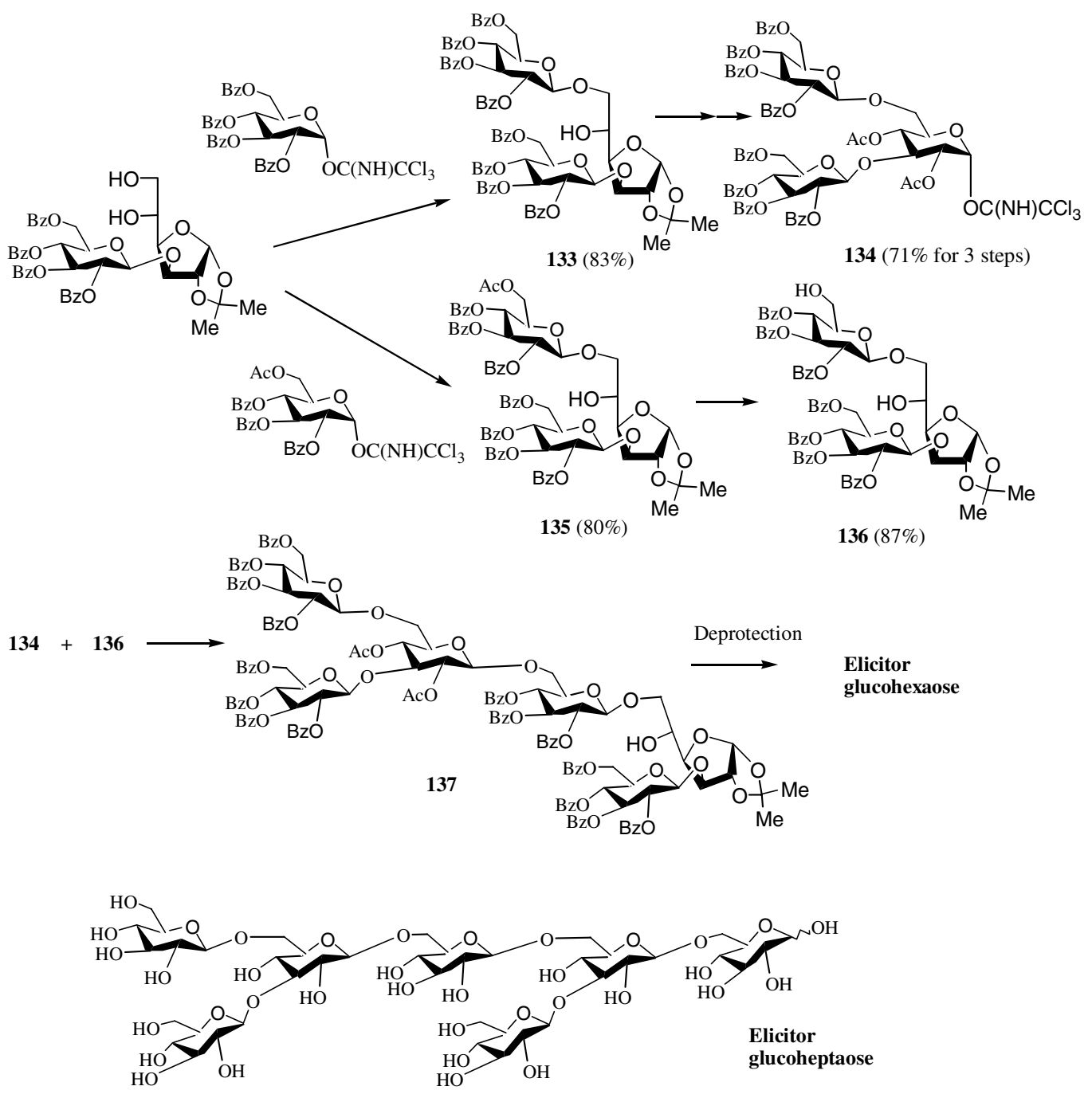

Scheme 27. Concise synthesis of elicitor glucohexaose.

GXM of Cryptococcus neoformans serotype C, reaction of a heptasaccharide acceptor 138 with methyl 2,3,4-tri$O$-acetyl- $\alpha$-D-glucopyranosyluronate trichloroacetimidate under the coupling conditions gave a neat sugarsugar orthoester 139. Rearrangement of $\mathbf{1 3 9}$ under normal conditions in the presence of TMSOTf $(0.02-$ $0.05 \%$ equiv) did not occur at all. Increment of the TMSOTf to 0.2 equiv and elevation of reaction temperature only caused decomposition. Meanwhile, no reaction between 138 and methyl 2,3,4-tri- $O$-acetyl- $\alpha$-Dglucopyranosyluronate bromide occurred. However, as reported in the previous work, ${ }^{24 \mathrm{~b}}$ a similar hexaoside acceptor 140 just lacking in the $\beta-(1 \rightarrow 4)$-Xyl $p$ branch at the middle mannose residue reacts with methyl 2,3,4-tri- $O$-acetyl- $\alpha$-D-glucopyranosyluronate bromide smoothly to give the heptasaccharide 141. It was hypothesized that the additional attached $(1 \rightarrow 4)$-Xyl $p$ in 138 changes the conformation of the acceptor $\mathbf{1 3 8}$ substantially making a serious steric hindrance at the
O-2 of the downstream mannose unit that inhibits the rearrangement (see Scheme 28).

\subsection{Rearrangement of sugar-sugar orthoesters leading to 1,2-cis glycosidic linkage}

Rearrangement of sugar-sugar orthoesters usually gives 1,2-trans-linked oligosaccharides as shown in Scheme 9. This is true for 6-linked sugar-sugar orthoesters and for 2-, 3-, or 6-linked mannose-mannose (or other sugar acceptor) orthoesters. Recent studies ${ }^{25 a-c}$ revealed that the rearrangement of 3-linked glucooligosaccharideglucooligosaccharide orthoesters sometimes gave a completely (or only) 1,2-cis glycosidic linkage.

3.7.1. Coupling of acylated $\beta-(1 \rightarrow 3)$-linked glucobiosyl trichloroacetimidate donor with $3^{\prime}$-unprotected acylated $\beta$ - $(1 \rightarrow 3)$-linked glucobiosyl acceptor gives pure $\alpha$-linked oligosaccharide. It was found ${ }^{25 a}$ that TMSOTf 

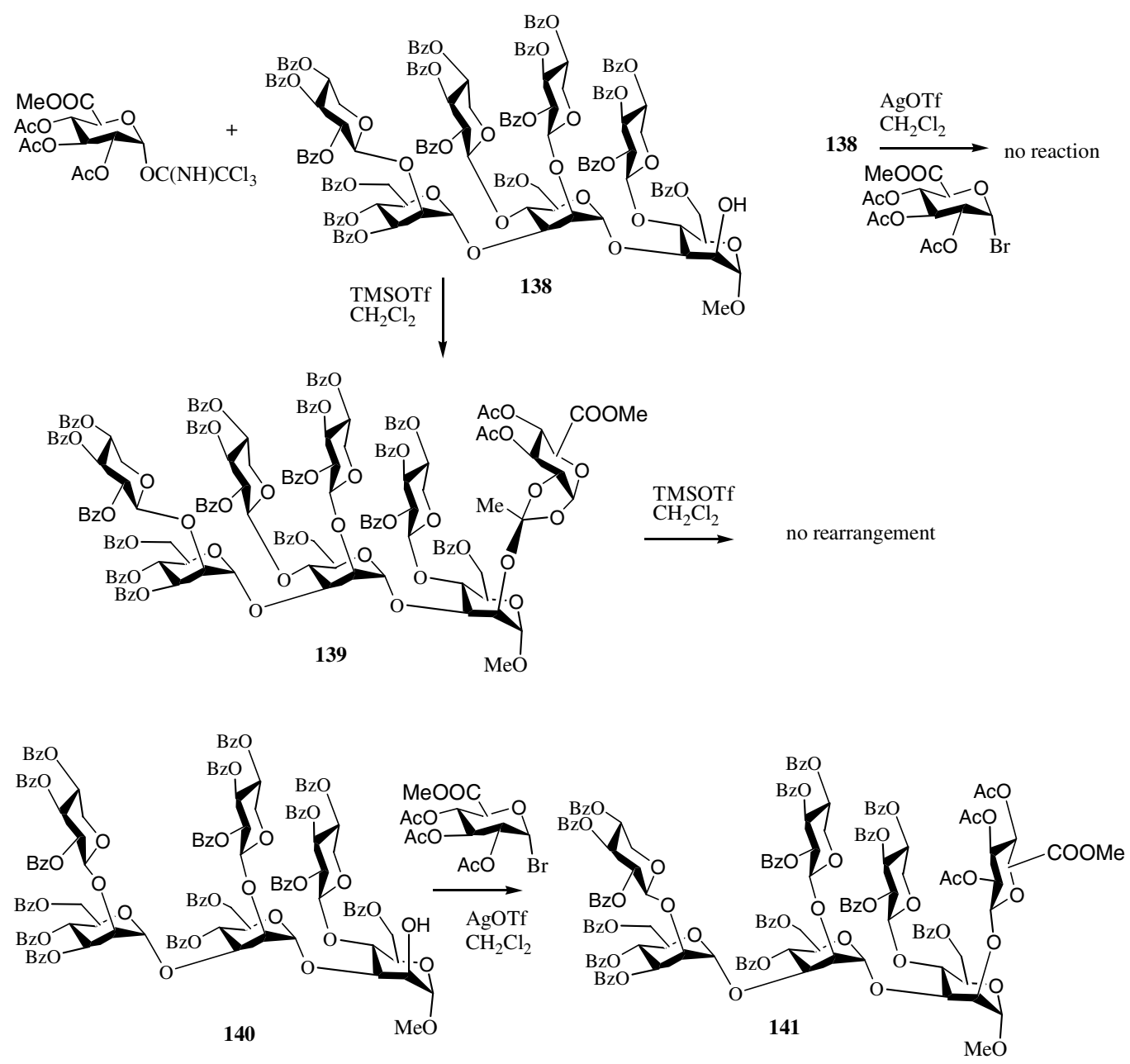

Scheme 28. Sugar-sugar orthoester, not rearranged.

catalyzed coupling of fully benzoylated $\beta-(1 \rightarrow 3)$-linked glucobiosyl trichloroacetimidate $\mathbf{1 4 2}$ with $3^{\prime}$-unprotected 4-methoxyphenyl benzoylated $\beta-(1 \rightarrow 3)$-linked glucobioside 143 produced pure $\alpha$-linked tetrasaccharide in spite of the presence of a C-2 ester capable of neighboring group participation. Also, TMSOTf catalyzed condensations of fully benzoylated glucosyl trichloroacetimidate with the disaccharide acceptor 143, and a $\beta$-(1 $\rightarrow 3)$-linked glucodiosyl trichloroacetimidate $\mathbf{1 4 4}$ with lauryl 2,4,6-tri- $O$-benzoyl- $\beta$-D-glucopyranoside yielded pure $\alpha$-linked trisaccharides as shown in Scheme 29 . The investigation ${ }^{25 b, \mathrm{c}}$ also revealed that coupling of $\alpha-(1 \rightarrow 3)$-linked glucodiosyl trichloroacetimidate $\mathbf{1 4 5}$ with a $3^{\prime}$-unprotected $\alpha-(1 \rightarrow 3)$-linked glucodioside acceptor 146 gave normal $\beta$ - $(1 \rightarrow 3)$-linked tetrasaccharide (Scheme 29) and the orthoester intermediate was detected on TLC at the initial stage of the reaction.

These observations indicate that in $(1 \rightarrow 3)$-glucosylation, the glycosyl bond originally present in either donor or acceptor control the stereoselectivity of the forthcoming bond, that is, the newly formed glycosidic linkage has the opposite anomeric configuration of that of either the donor or acceptor in spite of the presence of a C-2 ester group.

3.7.2. Sugar-sugar orthoester is the intermediate in $(1 \rightarrow 3)$-glucosylation capable of producing $\alpha$-linked oligosaccharide through intramolecular rearrangement. For investigation of the mechanism of $(1 \rightarrow 3)$-glucosylation leading to 1,2-cis linkages, the coupling reaction was slowed down to allow isolation of the intermediate. In the coupling of a disaccharide donor 147 with a trisaccharide acceptor 148, the orthoester intermediate 149 formed at the initial stage is separated and it was then converted to the $\alpha$-linked pentasaccharide $\mathbf{1 5 0}$ by treatment with catalytic TMSOTf. In the reaction of trisaccharide donor 134 with trisaccharide acceptor 151, the orthoester intermediate $\mathbf{1 5 2}$ was also captured, ${ }^{23 \mathrm{k}}$ and it was readily transformed to $\alpha$-linked hexasaccharide 153 in the presence of catalytic TMSOTf through an intramolecular rearrangement as indicated in Scheme 30 . The supposed mechanism ${ }^{25 a, 23 \mathrm{k}}$ involves $\mathrm{C}-1-\mathrm{O}-1$ bond cleavage by TMSOTf, and it is related to the pathway (a) from 2 to 4 in Chart 1. 

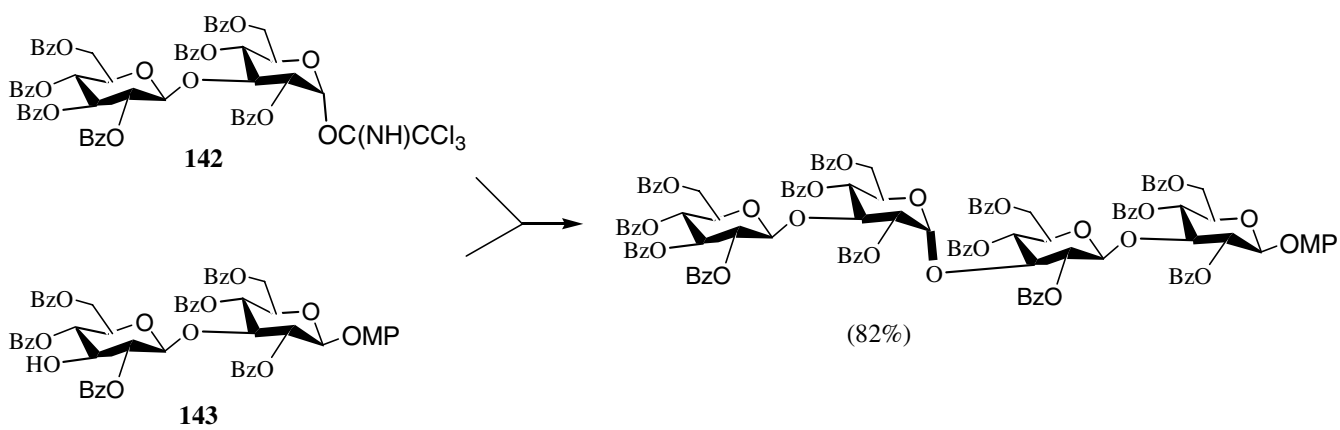

$(82 \%)$

143
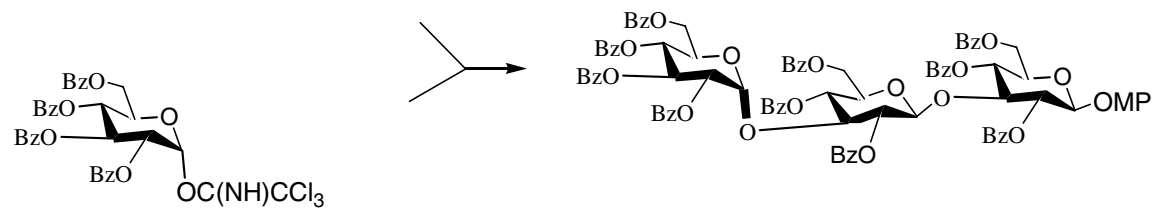

$(75 \%)$
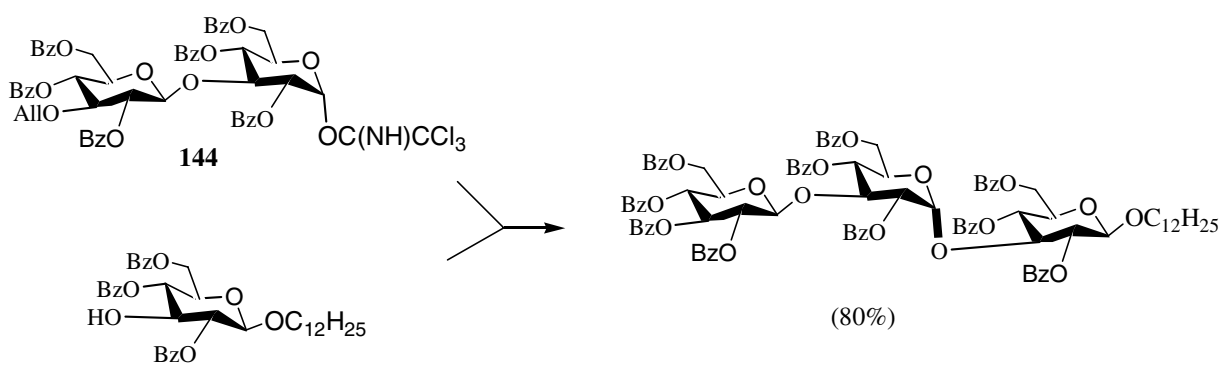

$(80 \%)$
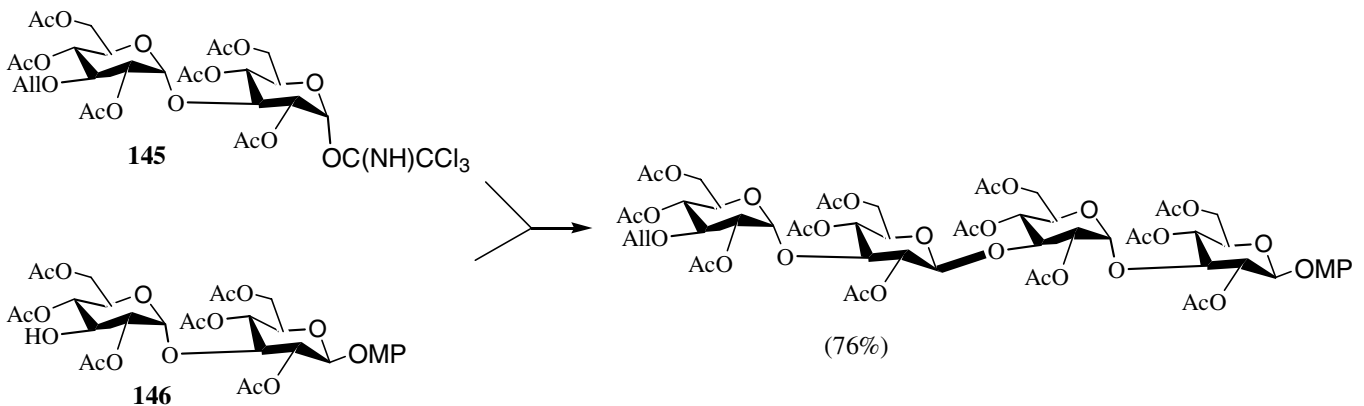

$(76 \%)$

Scheme 29. Abnormal and normal stereoselectivity in $(1 \rightarrow 3)$-glucosylation.

3.7.3. Application of the orthoester intramolecular rearrangement for the synthesis of novel glucans. Novel linear glucans with alternate $\alpha$ - and $\beta$ - $(1 \rightarrow 3)$-linkages ${ }^{25 d}$ were readily prepared through orthoester rearrangement with appropriate glucose mono- or disaccharide donors and acceptors. Scheme 31 shows glucohexa-, hepta-, and octaose thus synthesized.

Also, $\beta$ - $(1 \rightarrow 6)$-branched $(1 \rightarrow 3)$-glucononaose, ${ }^{25 e}$-glucododecaose, and -glucopentadecaose ${ }^{25 f}$ with alternate $\beta$ and $\alpha$-bonds in the backbone as shown in Scheme 32 were readily prepared in a similar way.

Similarly, the intramolecular couplings of nonasaccharide precursor 154 and dodecasaccharide precursor 155 produced the corresponding 6-branched cycloglu- cans $^{25 \mathrm{~g}} 156$ and 157 with alternate $\beta$ - and $\alpha-(1 \rightarrow 3)$ linked cycloglucohexose and cycloglucooctaose backbone, respectively, as shown in Scheme 33 .

\subsection{Summary}

The features of orthoesters discussed above can be summarized as follows. First, orthoesters composed of a sugar and a simple alcohol are not good glycosyl donors in oligosaccharide synthesis because of their multi-pathway reactions leading to relatively low yields. Second, formation of sugar-sugar orthoesters consisting of a fully acylated mono- or disaccharide donor and a partially protected mono- or disaccharide acceptor is 

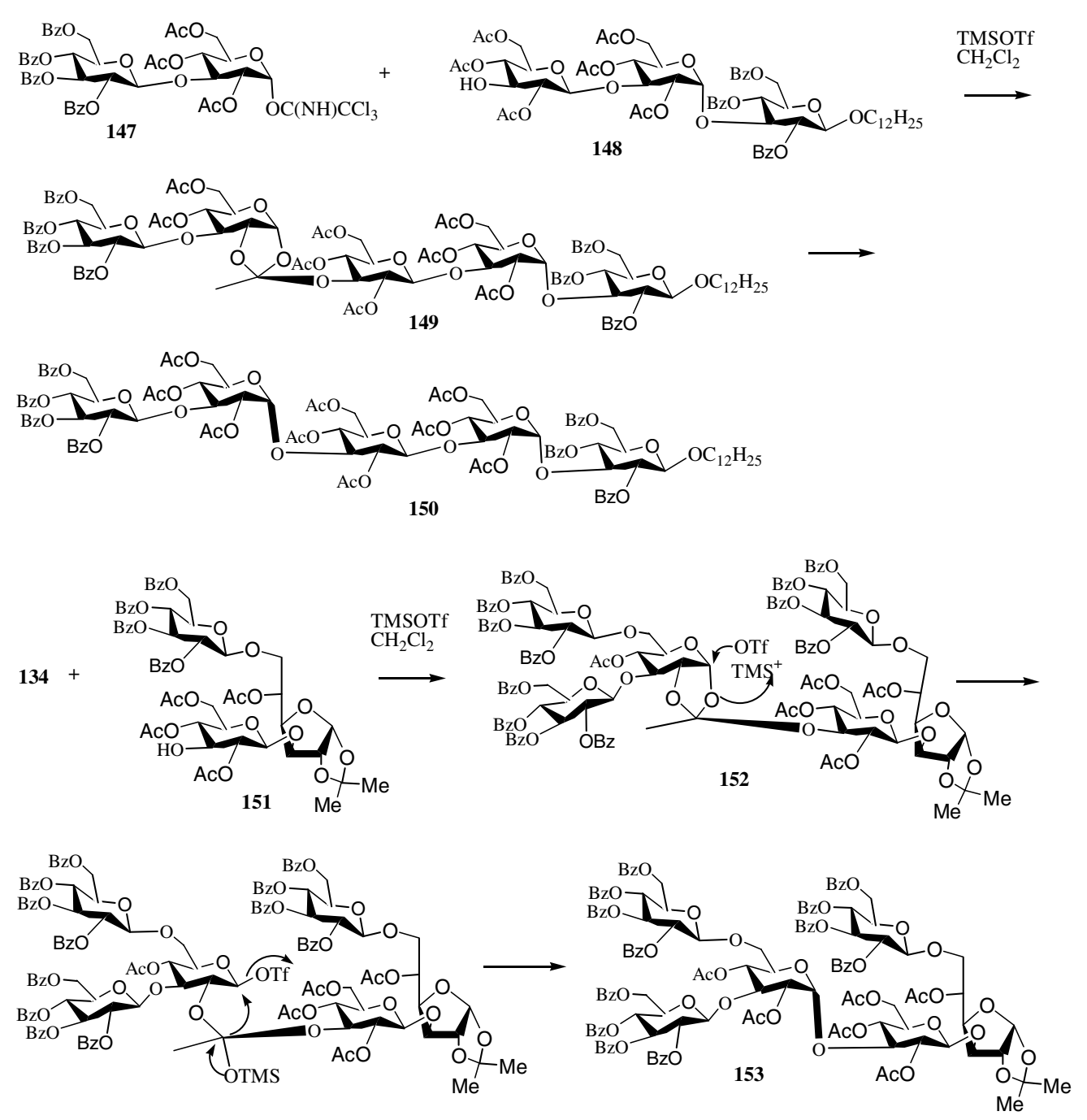

Scheme 30. Intramolecular rearrangement of orthoesters.

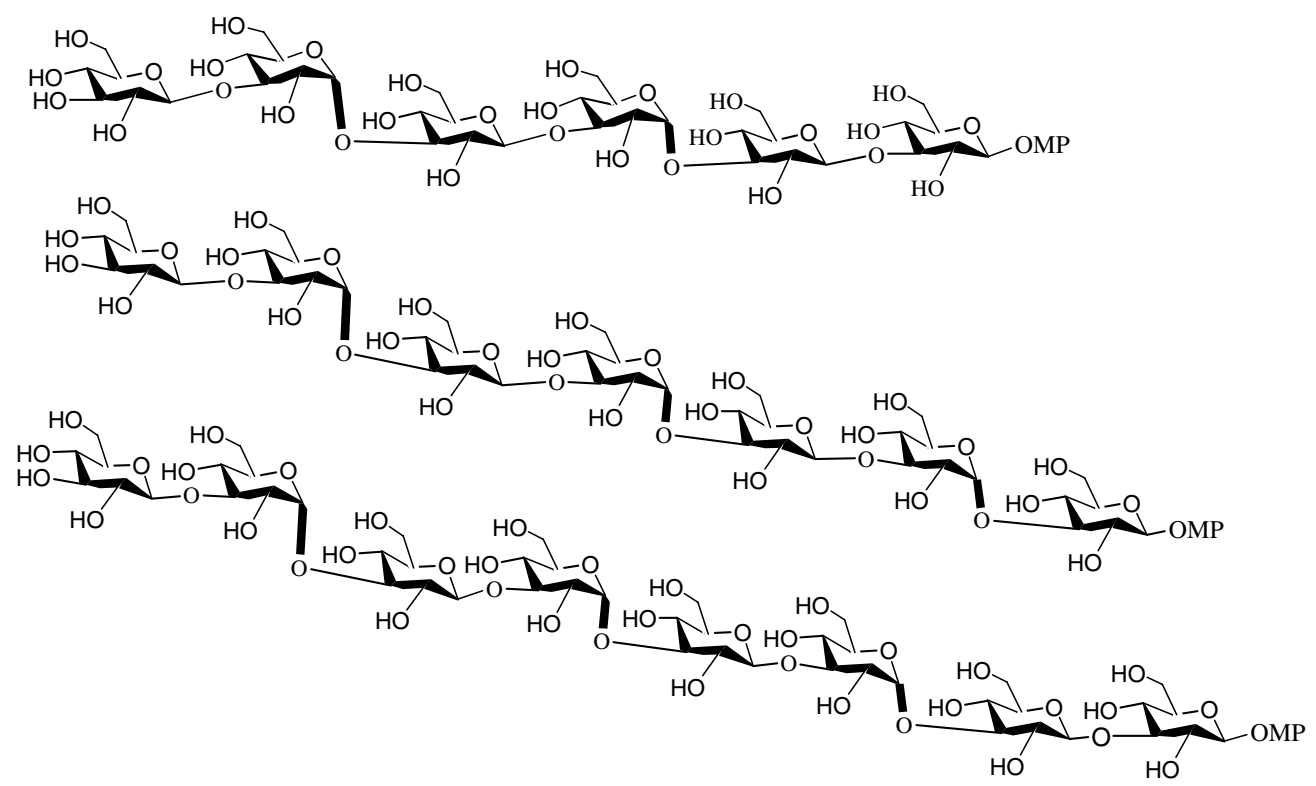

Scheme 31. Novel linear glucans with alternate $\alpha$ - and $\beta-(1 \rightarrow 3)$-linkages. 

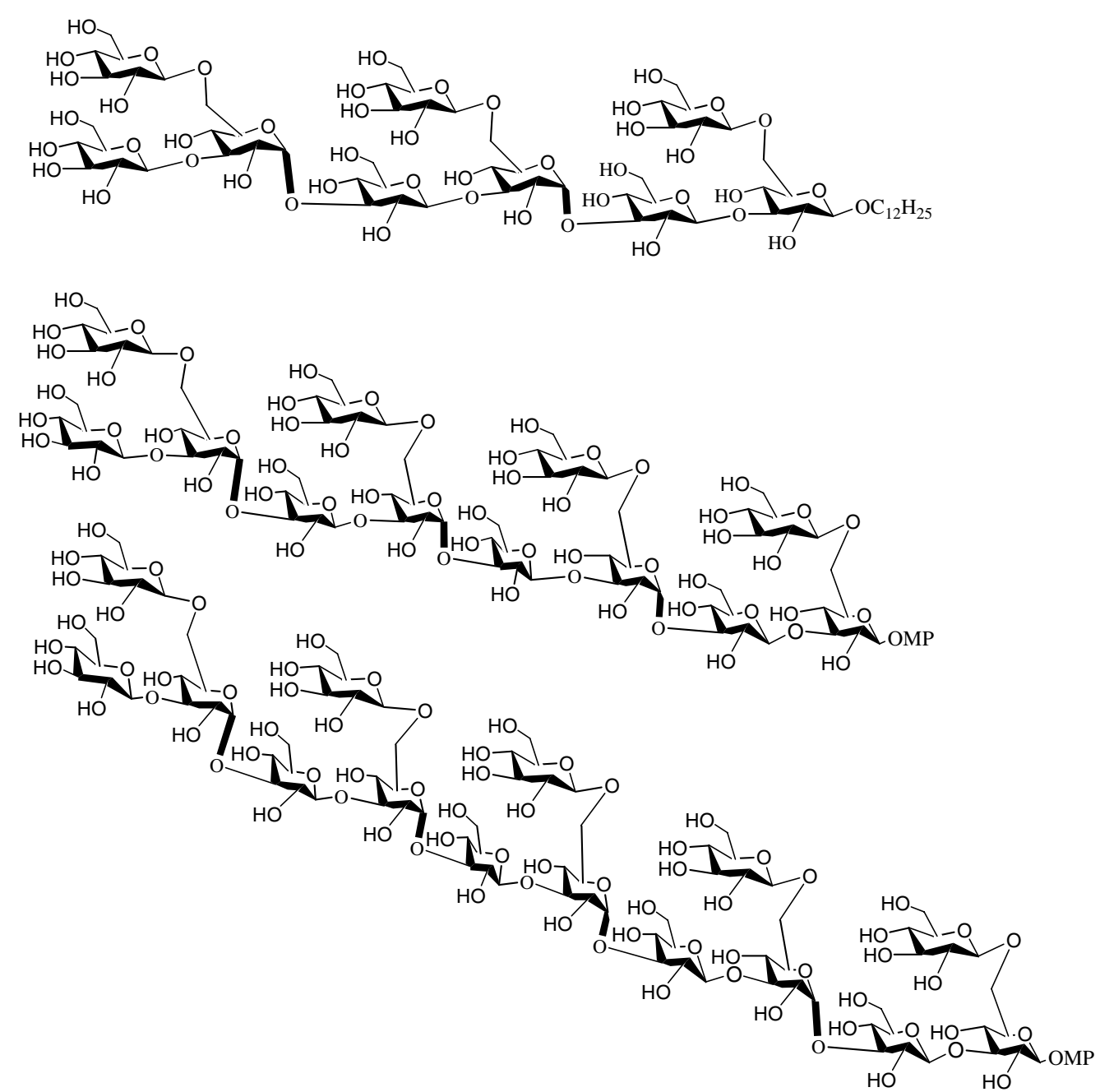

Scheme 32. Synthesized $\beta$-( $1 \rightarrow 6)$-branched $(1 \rightarrow 3)$-glucans with alternate $\beta$ - and $\alpha$-bonds in the backbone.
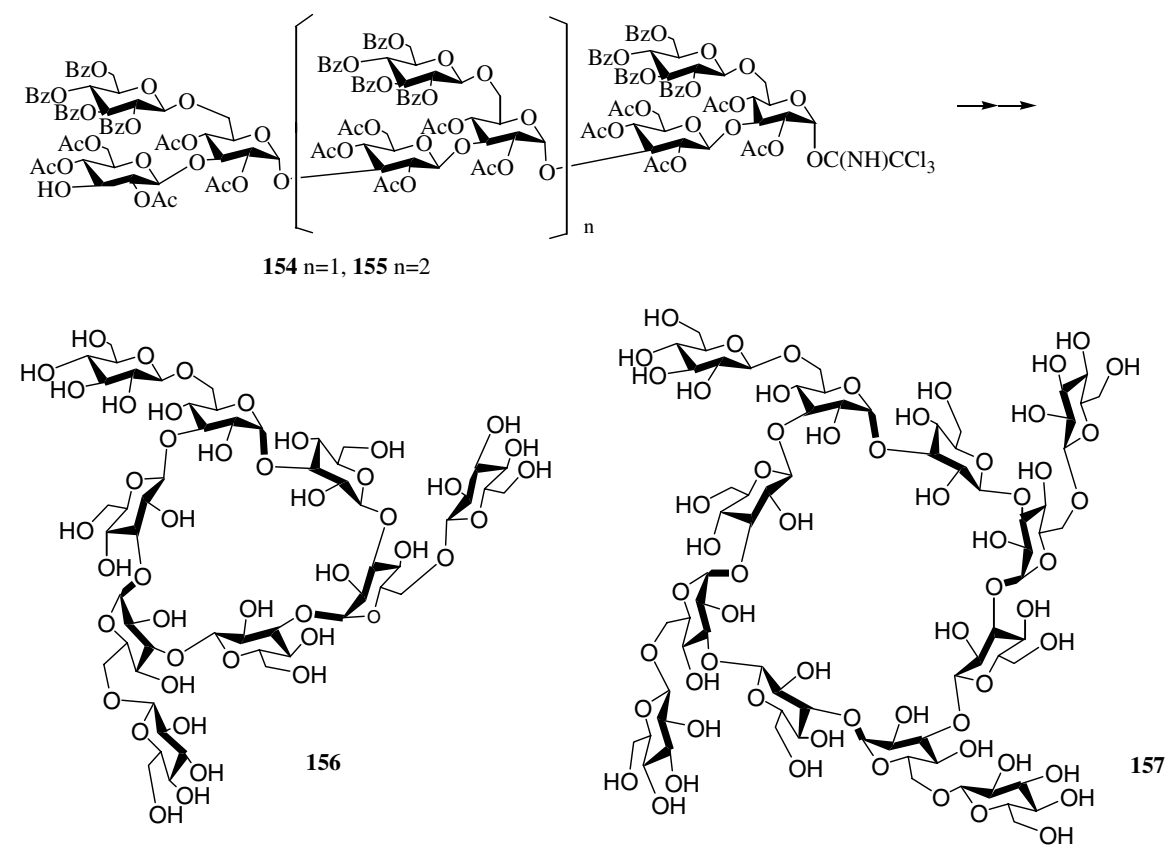

Scheme 33. Cycloglucans with alternate $\beta$ - and $\alpha-(1 \rightarrow 3)-$ linked backbones. 
regioselective and rearrangement of the orthoesters via $\mathrm{RO}-$ (orthoester)C bond cleavage gives a dioxolenium ion intermediate leading to 1,2-trans-glycosidic linkage. The activity order of hydroxyl groups in the partially protected mannose and glucose acceptors is $6-\mathrm{OH}>3$ $\mathrm{OH}>2-$ or $4-\mathrm{OH}$. Third, the coupling reactions with acylated glycosyl trichloroacetimidates as the donors usually give orthoesters as the intermediates specifically when the coupling is carried out at slowed rates, and this is successfully used in regio- and stereoselective syntheses of oligosaccharides. Fourth, mannose and rhamnose orthoesters readily undergo $\mathrm{O}-2$-(orthoester)C bond breaking, and this is used for synthesis of $\alpha-(1 \rightarrow 2)$ linked oligosaccharides. Fifth, $(1 \rightarrow 3)$-glucosylation is special since the rearrangement of sugar orthoester intermediates occurs with either RO-(orthoester)C bond cleavage with formation of the dioxolenium ion leading to 1,2-trans-linkage, or C-1-O-1 bond cleavage leading to 1,2-cis-linkage, and this is dependent upon the structure of the donor and acceptor that compose the orthoester.

There are still some unsolved problems, such as those encountere in $(1 \rightarrow 2)$ - and $(1 \rightarrow 4)$-glucosylation, and in $(1 \rightarrow 2)-$, $(1 \rightarrow 3)-$, and $(1 \rightarrow 4)$-galactosylation with acylated glycosyl trichloroacetimidate donors. It is unclear whether the sugar-sugar orthoesters are formed and, if so, what are their rearrangement pathways? Further research will answer these, and new findings may create new methods for synthesis of complex oligosaccharides.

\section{References}

1. Pacsu, E. Adv. Carbohydr. Chem 1945, 1, 77-95.

2. Kochetkov, N. K.; Bochkov, A. F.. In Recent Development in the Chemistry of Natural Carbon Compounds; Akademia Kiado: Budapest, 1972; Vol. 4, pp 77-91.

3. (a) Kochetkov, N. K.; Khorlin, A. J.; Bochkov, A. F. Tetrahedron Lett. 1964, 289-292; (b) Kochetkov, N. K.; Khorlin, A. J.; Bochkov, A. F. . Tetrahedron 1967, 23, 693-707.

4. (a) Lemieux, R. U. Chem. Can. 1964, 10, 14-22; (b) Snyatkova, V. I.; Kochetkov, N. K. Izv. Akad. Nauk SSSR Ser. Khim 1969, 5, 1143-1149; (c) Cordes, E. H. Prog. Phys. Org. Chem. 1967, 4, 1-15; (d) Banoub, J.; Bundle, D. R. Can. J. Chem. 1979, 57, 2091-2097; (e) Bochkov, A. F.; Zaikov, G. E. The Chemistry of the $O$ Glycosidic Bond; Pergamon Press: New York, 1979, p 210; (f) Kochetkov, N. K.; Bochkov, A. F.; Sokolovskaya, T. A.; Snyatkova, V. I. Carbohydr. Res. 1971, 16, 17-27; (g) Bochkov, A. F.; Betanely, V. I.; Kochetkov, N. K. Carbohydr. Res. 1973, 30, 418-419; (h) Gorin, P. A. J. Carbohydr. Res. 1982, 101, 13-20.

5. (a) Hickenbottom, W. J. J. Chem. Soc. 1928, 3140-3144; (b) Perlin, A. S. Can. J. Chem. 1963, 41, 555-563; (c) Franks, N. E.; Montgomery, R. Carbohydr. Res. 1967, 3, 511-517; (d) Franks, N. E.; Montgomery, R. Carbohydr. Res. 1968, 6, 286-291; (e) Lindhorst, T. K. J. Carbohydr. Chem. 1997, 16, 237-241.
6. Schmidt, R. R.; Kinzy, W. Adv. Carbohydr. Chem. Biochem. 1994, 50, 21-125.

7. Toshima, K.; Tatsuta, K. Chem. Rev. 1993, 93, 1503-1542.

8. Kochetkov, N. K. Tetrahedron 1987, 2389-2436.

9. (a) Backinowski, L. V.; Nifant'ev, N. E.; Kochetkov, N. K. Bioorg. Khim. (USSR) 1983, 9, 1089-1095; (b) Backinowsky, L. V.; Nifantiev, N. E.; Kochetkov, N. K. Bioorg. Khim. (USSR) 1984, 10, 226-231; (c) Kochetkov, N. K.; Malysheva, N. N.; Klimov, E. M. Bioorg. Khim. (USSR) 1985, 11, 391-396.

10. (a) Bochkov, A. F.; Obruchnikov, I. V.; Kalinevich, V. M.; Kochetkov, N. K. Tetrahedron Lett. 1975, 3403-3406; (b) Kochetkov, N. K.; Ott, A. Y. Izv. Akad. Nauk SSSR, Ser. Khim. 1984, 2358-2365; (c) Backinowsky, L. V.; Oseledchik, T. A.; Kochetkov, N. K. Izv. Akad. Nauk SSSR, Ser. Khim. 1981, 1387-1393.

11. Kochetkov, N. K.; Klimov, E. M. Tetrahedron Lett. 1981, 337-340.

12. (a) Zurabyan, S. E.; Tikhomirov, M. M.; Nesmeyanov, V. A.; Khorlin, A. Y. Carbohydr. Res. 1973, 26, 117-123; (b) Wulff, G.; Kruger, W. Carbohydr. Res. 1971, 19, 139-142; (c) Dahlin, B.; Garegg, P. J.; Johansson, R.; Sammuelsson, B.; Orn, U. Acta Chem. Scand. B 1981, 35, 669-670.

13. (a) Ogawa, T.; Matsui, M. Carbohydr. Res. 1976, 51, C13C18; (b) Banoub, J.; Boullanger, P.; Potier, M.; Descotes, G. Tetrahedron Lett. 1986, 4145-4148; (c) Sznaidman, M. L.; Johnson, S. C.; Crasto, G.; Hecht, S. M. J. Org. Chem. 1995, 60, 3942-3943; (d) Wang, W.; Kong, F. Carbohydr. Res. 1995, 319, 117-127; (e) Wang, W.; Kong, F. J. Carbohydr. Chem. 1999, 18, 263-270.

14. (a) Ogawa, T.; Beppu, K.; Nakabayachi, M. Carbohydr. Res. 1981, 93, C6-C9; (b) Gass, J.; Strobl, M.; Loibner, A.; Kosma, P.; Zahringer, U. Carbohydr. Res. 1993, 244, 69-84; (c) Wang, W.; Kong, F. Tetrahedron Lett. 1998, 1937-1940.

15. (a) Kanie, O.; Hindsgaul, O. Curr. Opin. Struct. Biol. 1992, 2, 674-682; (b) Becker, B.; Furneaux, R. H.; Reck, F.; Zubkov, O. A. Carbohydr. Res. 1999, 315, 148-154; (c) Kaur, K. J.; Hindsgaul, O. Glycoconjugate J. 1991, 8, 9098; (d) Figueroa-Perez, S.; Verez-Bencomo, V. J. Carbohydr. Chem. 1998, 17, 851-861.

16. (a) Wang, W.; Kong, F. J. Org. Chem. 1998, 63, 5744 5745; (b) Wang, W.; Kong, F. Tetrahedron Lett. 1998, 1937-1940.

17. Wang, W.; Kong, F. Angew. Chem., Int. Ed. 1999, 38, $1247-1250$

18. Du, Y.; Kong, F. J. Carbohydr. Chem. 1999, 18, 655664.

19. (a) Wang, W.; Kong, F. Synth. Commun. 1999, 29, 3179 3184; (b) Wang, W.; Kong, F. J. Org. Chem. 1999, 64 5091-5095; (c) Zhu, Y.; Kong, F. J. Carbohydr. Chem. 2000, 19, 837-848; (d) Byramova, N. E.; Ovchinnikov, M. V.; Backinowsky, L. V.; Kochetkov, N. K. Carbohydr. Res. 1983, 124, c8-c11; (e) Wang, W.; Kong, F. Tetrahedron Lett. 1999, 1360-1363.

20. (a) Zhu, Y.; Kong, F. Synlett 2000, 1783-1787; (b) Zhu, Y.; Kong, F. Chin. J. Chem. 2001, 19, 120-122; (c) Zhu, Y.; Chen, L.; Kong, F. Chin. J. Chem. 2001, 19, 12891295; (d) Zhu, Y.; Chen, L.; Kong, F. Synth. Commun. 2002, 38, 1219-1226; (e) Chen, L.; Zhu, Y.; Kong, F. Carbohydr. Res. 2002, 337, 383-390.

21. (a) Zhu, Y.; Kong, F. Synlett 2001, 1217-1220; (b) Zhu, Y.; Chen, L.; Kong, F. Carbohydr. Res. 2002, 337, 207215; (c) Zhang, J.; Ma, Z.; Kong, F. Carbohydr. Res. 2003, 338, 2039-2046; (d) Zeng, Y.; Zhang, J.; Kong, F. Carbohydr. Res. 2002, 337, 1367-1371; (e) Ma, Z.; Zhang, J.; Kong, F. Tetrahedron: Asymmetry 2004, 15, 1517-1525; 
(f) Zhang, J.; Ma, Z.; Kong, F. Carbohydr. Res. 2003, 338, 1711-1718; (g) Ma, Z.; Zhang, J.; Kong, F. Carbohydr. Res. 2004, 339, 29-35; (h) Xing, Y.; Ning, J. Tetrahedron: Asymmetry 2003, 14, 1275-1283.

22. (a) Han, X.-B.; Jiang, Z.-H.; Schmidt, R. R. Liebigs Ann. Chem. 1993, 853-862; (b) Zhu, Y.; Kong, F. Synlett 2000, 663-667; (c) Zhu, Y.; Kong, F. Carbohydr. Res. 2001, 332, $1-21$.

23. (a) Kong, F. Curr. Org. Chem. 2003, 7, 841-865; (b) Zhang, J.; Kong, F. Tetrahedron: Asymmetry 2002, 13, 243-252; (c) Ning, J.; Heng, L.; Kong, F. Tetrahedron Lett. 2002, 673-676; (d) Heng, L.; Ning, J.; Kong, F. J. Carbohydr. Chem. 2001, 20, 285-294; (e) Zhang, J.; Kong, F. J. Carbohydr. Chem. 2002, 21, 79-86; (f) Zhang, J.; Zhu, Y.; Kong, F. Carbohydr. Res. 2002, 337, 391-396; (g) Zhang, J.; Ning, J.; Kong, F. Carbohydr. Res. 2003, 338, 1023-1032; (h) Zhang, J.; Kong, F. Tetrahedron 2003, 59, 1429-1437; (i) Yang, G.; Kong, F. Synlett 2000, 1423-
1428; (j) Yang, G.; Kong, F. Carbohydr. Res. 2005, 340, 39-48; (k) Ning, J.; Zhang, W.; Yi, Y.; Yang, G.; Wu, Z.; Yi, J.; Kong, F. Bioorg. Med. Chem. 2003, 11, 2193-2203; (1) Ning, J.; Yi, Y.; Kong, F. Tetrahedron Lett. 2002, 43, 5545-5548; (m) Ning, J.; Kong, F.; Lin, B.; Lei, H. J. Agric. Food Chem. 2003, 51, 987-991; (n) Yi, Y.; Zhou, Z.; Ning, J.; Kong, F.; Li, J. Synthesis 2003, 4, 491-496.

24. (a) Zhao, W.; Kong, F. Carbohydr. Res. 2005, 340, 16731681; (b) Zhao, W.; Kong, F. Bioorg. Med. Chem. 2005, 13, 121-130.

25. (a) Zeng, Y.; Ning, J.; Kong, F. Tetrahedron Lett. 2002, 3729-3733; (b) Zeng, Y.; Ning, J.; Kong, F. Chin. J. Chem. 2002, 20, 1142-1144; (c) Zeng, Y.; Ning, J.; Kong, F. Carbohydr. Res. 2003, 338, 307-311; (d) Zeng, Y.; Kong, F. Carbohydr. Res. 2003, 338, 2047-2056; (e) Wu, Z.; Ning, J.; Kong, F. Chin. J. Chem. 2003, 21, 1655-1660; (f) Wu, Z.; Ning, J.; Kong, F. Carbohydr. Res. 2003, 338, 2203-2212; (g) Wu, Z.; Kong, F. Synlett 2004, 2594-2596. 